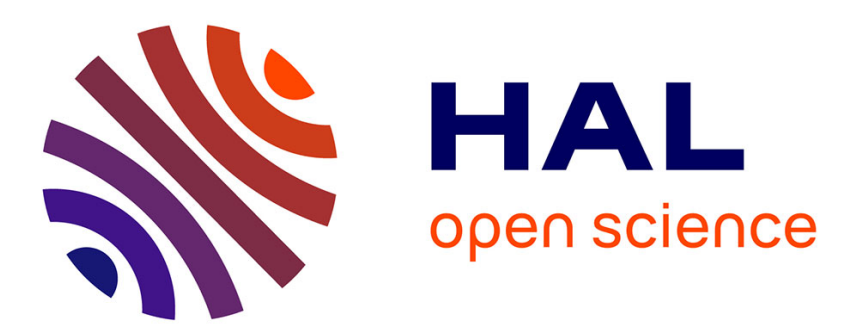

\title{
Experimental study of the effect of sodium chloride on drying of porous media: The crusty-patchy efflorescence transition
}

Houda Eloukabi, Nour Sghaier-Ben Chiekh, Sassi Ben Nasrallah, Marc Prat

\section{- To cite this version:}

Houda Eloukabi, Nour Sghaier-Ben Chiekh, Sassi Ben Nasrallah, Marc Prat. Experimental study of the effect of sodium chloride on drying of porous media: The crusty-patchy efflorescence transition. International Journal of Heat and Mass Transfer, 2013, vol. 56, pp. 80-93. 10.1016/j.ijheatmasstransfer.2012.09.045 . hal-00951512

\section{HAL Id: hal-00951512 \\ https://hal.science/hal-00951512}

Submitted on 24 Feb 2014

HAL is a multi-disciplinary open access archive for the deposit and dissemination of scientific research documents, whether they are published or not. The documents may come from teaching and research institutions in France or abroad, or from public or private research centers.
L'archive ouverte pluridisciplinaire HAL, est destinée au dépôt et à la diffusion de documents scientifiques de niveau recherche, publiés ou non, émanant des établissements d'enseignement et de recherche français ou étrangers, des laboratoires publics ou privés. 


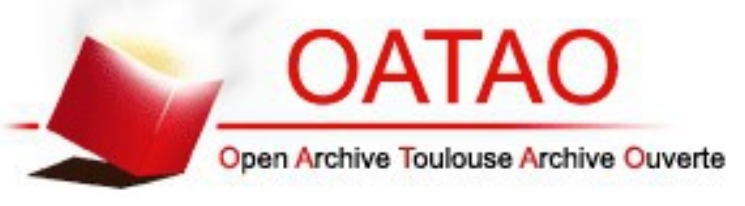

\section{Open Archive TOULOUSE Archive Ouverte (OATAO)}

OATAO is an open access repository that collects the work of Toulouse researchers and makes it freely available over the web where possible.

This is an author-deposited version published in : http://oatao.univ-toulouse.fr/ Eprints ID : 11022

To link to this article : DOI:10.1016/j.ijheatmasstransfer.2012.09.045

URL : http://dx.doi.org/10.1016/j.ijheatmasstransfer.2012.09.045

To cite this version : Eloukabi, Houda and Sghaier-Ben Chiekh, Nour and Ben Nasrallah, Sassi and Prat, Marc Experimental study of the effect of sodium chloride on drying of porous media: The crustypatchy efflorescence transition. (2013) International Journal of Heat and Mass Transfer, vol. 56 ( $\left.{ }^{\circ} 1-2\right)$. pp. 80-93. ISSN 0017-9310

Any correspondance concerning this service should be sent to the repository administrator: staff-oatao@listes-diff.inp-toulouse.fr 


\title{
Experimental study of the effect of sodium chloride on drying of porous media: The crusty-patchy efflorescence transition
}

\author{
H. Eloukabi ${ }^{\text {a }}$ N. Sghaier ${ }^{\text {a }}$, S. Ben Nasrallah ${ }^{\text {a }}$, M. Prat ${ }^{\text {b,c,* }}$ \\ ${ }^{a}$ Laboratoire d'Eudes des Systèmes Thermiques et Energétiques de Monastir, École nationale d'Ingénieurs de Monastir, 5000 Monastir, Tunisia \\ ${ }^{\mathrm{b}}$ INPT, UPS, IMFT (Institut de Mécanique des Fluides de Toulouse), Université de Toulouse, Allée Camille Soula, F-31400 Toulouse, France \\ ${ }^{\mathrm{c}} \mathrm{CNRS}$, IMFT, F-31400 Toulouse, France
}

Keywords:

Drying

Porous medium

Salt crystallization

Efflorescence

\begin{abstract}
A B S T R A C T
We present an experimental study of drying in the presence of dissolved sodium chloride. The process is characterized by the formation of a crystallized salt layer, referred to as efflorescence, at the evaporative surface of the porous medium. By varying the average size of the beads forming the porous medium, we show that the formation of the crystal layer does not affect significantly the drying process and can even enhance the drying rate when the beads are sufficiently large. By contrast, the crystal layer can greatly affect the drying process and even blocks or severely limit the evaporation process for sufficiently small beads. We therefore show the existence of two regimes, namely the blocking regime and the enhanced drying rate regime. It is shown that the two regimes correspond to two different types of efflorescence, referred to as crusty and patchy, respectively. Then by varying the initial salt concentration for a given bead size, we show that the interplay between drying and the efflorescence formation leads to a nonmonotonous variation of the drying rate with the initial salt concentration when the efflorescence is patchy but not when the efflorescence is crusty. The crusty-patchy transition is finally discussed from a simple model of capillary rise in the efflorescence.
\end{abstract}

\section{Introduction}

Evaporation in porous media in the presence of dissolved salt is of interest in relation with at least three important applications: soil physics and related issues, e.g. [1] and references therein, injection of $\mathrm{CO}_{2}$ in aquifer [2] and civil engineering owing to the major damages that can be induced by the salt crystallization process, e.g. $[3,4]$. Despite the importance of these various fields, the impact of the presence of salt on the evaporation process is still poorly understood. This is mainly so because the evaporation process in the presence of salt usually leads to the formation of salt crystals and to complex interactions between the crystallized regions and the various transport phenomena involved in the process. The crystals can form at the surface of the porous medium and are then called efflorescence or within the porous medium, where they form subflorescence. In the present paper, we will consider sodium chloride as dissolved salt, a salt known for its tendency to form efflorescence [5]. As explained in several previous papers, e.g. [6-8], the equation governing the dissolved salt transport with the porous structure during drying is a transient convec-

* Corresponding author at: INPT, UPS, IMFT (Institut de Mécanique des Fluides de Toulouse), Université de Toulouse, Allée Camille Soula, F-31400 Toulouse, France.

E-mail address: mprat@imft.fr (M. Prat). tion-diffusion equation. Under usual evaporation conditions the convection effect is sufficient to induce greater concentration at the porous surface, which therefore is the expected location of crystal formation since the salt crystal begins to form when the concentration reaches a sufficiently high concentration. In the case of sodium chloride, this crystallization concentration is expected to be the saturation concentration since supersaturation effects are usually negligible with $\mathrm{NaCl}[9,10]$.

According to the available literature, the effect on efflorescence formation on drying process could appear as a controversial issue. For example, several studies, e.g. $[1,11]$ and references therein, report that the efflorescence forms a crust, whose net effect is to severely limit the evaporation. On the other hand, other studies [1216] do not report a significant blocking effect, at least as long as the efflorescence is hydraulically connected to the aqueous solution present within the porous medium. On the contrary, it is even observed that the efflorescence formation can even enhance the drying rate $[12,13]$. Furthermore, there are also important differences in the analysis of the mechanisms controlling the efflorescence growth. In [12-16], the efflorescence is seen as a set of isolated porous structures saturated by the aqueous solution and growing as a result of salt precipitation at the outer surface of efflorescence structures where evaporation takes place. Growth stops when the efflorescence ceases to be hydraulically connected to the aqueous 


\begin{tabular}{|c|c|c|c|}
\hline \multicolumn{4}{|c|}{ Nomenclature } \\
\hline$a$ & mean distance between patches (m) & $P_{\text {atm }}$ & atmospheric pressure $(\mathrm{Pa})$ \\
\hline$a_{\mathrm{w}}$ & water activity & $P_{\text {ce }}$ & capillary pressure on top of the efflorescence structure \\
\hline $\begin{array}{l}A \\
b\end{array}$ & top surface area $\left(\mathrm{m}^{2}\right)$ & & $(\mathrm{Pa})$ \\
\hline$B$ & $\begin{array}{l}\text { numerical factor } \\
\text { Bond number }\end{array}$ & $P_{\mathrm{cpm}}$ & capillary pressure of the porous medium (Pa) \\
\hline$c$ & $\begin{array}{l}\text { Bond number } \\
\text { numerical factor }\end{array}$ & $\mathrm{Pe}$ & Peclet number \\
\hline C & $\begin{array}{l}\text { numerical factor } \\
\text { dissolved salt mass fraction (\%) }\end{array}$ & $P_{\mathrm{e}}\left(h_{\mathrm{e}}\right)$ & pressure in the liquid at the top of the efflorescence \\
\hline $\mathrm{Ca}$ & $\begin{array}{l}\text { dissolved sait mass fraction (\%) } \\
\text { capillary number }\end{array}$ & & structure (Pd) \\
\hline$C_{\text {sat }}$ & $\begin{array}{l}\text { capillary number } \\
\text { saturation mass fraction (\%) }\end{array}$ & $P_{\mathrm{e}}(0)$ & pressure in the liquid at the bottom of the efflorescence \\
\hline$C_{0}$ & $\begin{array}{l}\text { Saturation mass Iraction (\%) } \\
\text { dissolved salt initial mass fraction (\%) }\end{array}$ & 0 & Structure (Pa) \\
\hline$d$ & bead diameter $(\mathrm{m})$ & Q & 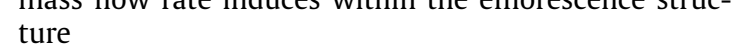 \\
\hline$d_{\mathrm{e}}$ & diameter of efflorescence structure (m) & $r$ & radial distance from the middle of the porous surface \\
\hline$D_{\mathrm{s}}$ & salt molecular diffusion coefficient $\left(\mathrm{m}^{2} / \mathrm{s}\right)$ & $r_{\mathrm{c}}$ & radius of the cylinder \\
\hline$D_{\mathrm{s}}^{*}$ & salt effective diffusion coefficient $\left(\mathrm{m}^{2} / \mathrm{s}\right)$ & $R H_{\infty}$ & relative humidity (\%) \\
\hline$F^{s}$ & fraction of water evaporated from the sample at the end & $S$ & liquid saturation \\
\hline & of drying & $t$ & time $(\mathrm{s})$ \\
\hline$g$ & gravitational acceleration $\left(\mathrm{m} / \mathrm{s}^{2}\right)$ & $T$ & temperature $(\mathrm{K})$ \\
\hline$h_{\mathrm{e}}$ & height of efflorescence structure (m) & $U$ & average interstitial velocity of the solution $(\mathrm{m} / \mathrm{s})$ \\
\hline$j$ & evaporation flux $\left(\mathrm{kg} / \mathrm{m}^{2} / \mathrm{s}\right)$ & $U_{\mathrm{z}}$ & velocity component normal to the porous medium sur- \\
\hline$j_{\mathrm{e}}$ & evaporation flux at the top of efflorescence structure & & face $(\mathrm{m} / \mathrm{s})$ \\
\hline & $\left(\mathrm{kg} / \mathrm{m}^{2} / \mathrm{s}\right)$ & $V$ & volume of water \\
\hline$j_{0}$ & prefactor & $V_{\mathrm{p}}$ & volume of the pore space within the porous medium \\
\hline$J$ & evaporation rate $\left(\mathrm{kg} / \mathrm{m}^{2} / \mathrm{s}\right)$ & & \\
\hline$K_{\mathrm{e}}$ & permeability of salt structure $\left(\mathrm{m}^{2}\right)$ & \multicolumn{2}{|c|}{ Greek symbols } \\
\hline$L$ & height of the porous sample $(\mathrm{m})$ & $\delta_{\mathrm{e}}$ & efflorescence mean pore size $(\mathrm{m})$ \\
\hline$m_{\text {salt }}$ & mass of salt $(\mathrm{g})$ & $\varepsilon$ & porosity of the porous medium \\
\hline$m_{\mathrm{w}}$ & the total mass of liquid evaporated at the end of drying & $\varepsilon_{\mathrm{e}}$ & porosity of salt structure \\
\hline & (g) & & solution viscosity (Pa s) \\
\hline$m_{\mathrm{w} 0}$ & mass of liquid water present initially in the system (g) & $\rho$ & solution density $\left(\mathrm{kg} / \mathrm{m}^{3}\right)$ \\
\hline & unit vector normal to the considered surface & $\sigma$ & surface tension $(\mathrm{N} / \mathrm{m})$ \\
\hline$p_{\mathrm{v}}(C)$ & vapor pressure for a $\mathrm{NaCl}$ aqueous solution $(\mathrm{Pa})$ & $\tau$ & dimensionless crystallization time \\
\hline & vapor pressure for pure water $(\mathrm{Pa})$ & & \\
\hline$p_{\mathrm{v} \infty}$ & vapor pressure in the regulated chamber $(\mathrm{Pa})$ & & \\
\hline
\end{tabular}

solution in the porous medium. This analysis is supported by imbibition experiments on dry efflorescence, which indeed show that the efflorescence is porous, X-ray tomography images, which confirm the internal porous nature of efflorescence, and visualization experiments with micromodels, which allows to see accurately when the efflorescence ceases to be connected to the underlying aqueous solution. Although not directly supported by experimental evidences, a completely different analysis was proposed in [11], with a strong emphasis on transport of water in vapor phase due to salt hygroscopic effects. According to this analysis, the efflorescence would grow from salt precipitation at its bottom (porous medium/efflorescence interface) rather than precipitation at the efflorescence outer surface. This analysis is not consistent with the experiments reported in [12-15] but might be relevant in the case of blocking crust formation, which is the case considered in [11]. This short review clearly illustrates that much remains to be done to fully understand the factors controlling the formation, growth and properties of efflorescence.

In this context, the present experimental study brings two new elements, which illustrate the complex interactions induced by the efflorescence formation. First, it is shown that both the blocking crust formation and the enhanced drying rate efflorescence can form under about the same evaporation condition simply by changing the average pore size of the medium. This leads to identify two main types of efflorescence referred to as crusty (= blocking) and patchy, respectively. Second, it is shown that the mean evaporation rate in the first phase of drying, where the efflorescence is hydraulically connected to the underlying aqueous solution, varies very differently with the initial salt concentration depending on the type of efflorescence. The mean evaporation rate increases monotonously with the initial concentration when the efflorescence is crusty whereas the variation is non-monotonous when the efflorescence is patchy. Since the activity of the solution is a decreasing function of the salt concentration, this non-monotonous variation is attributed to the complex interplay between the evaporation process and the efflorescence formation and growth.

The paper is organized as follows. The experimental set-up is described in Section 2. Then we present and discuss the results obtained by varying the average pore size under similar evaporation condition. Results on the influence of initial salt concentration are also presented in this section. A simple phenomenological model of efflorescence is proposed in Section 4 . We close the article by offering a conclusion in which we summarize the main findings.

\section{Experimental set-up}

Drying experiments are performed for porous samples consisting of packing of approximately spherical glass beads initially saturated with a $\mathrm{NaCl}$ aqueous solution. Six ranges of bead diameters were used $(5-50 \mu \mathrm{m}),(50-62 \mu \mathrm{m}),(100-160 \mu \mathrm{m}),(200-250 \mu \mathrm{m})$, (250-270 $\mu \mathrm{m}), 300 \mu \mathrm{m}$. These various ranges are referred to as range \#1, \#2, \#3, \#4, \#5 and \#6, respectively.

A sample is prepared by mixing the beads with a sodium-chloride aqueous solution of given concentration in a container to obtain a saturated viscous paste. Then this paste is poured in a cylindrical vessel (diameter $2 \mathrm{~cm}$; depth $1 \mathrm{~cm}$ ). The vessel is 
machined in a Plexiglas cylinder. Plexiglas has been chosen because of the relatively high contact angle of the solution on the order of $80^{\circ}$ independently of the salt concentration [17]. Together with a smooth machining of inner wall, this high contact angle prevents the formation of parasitic wetting films on the vessel walls. The packing is left for settling for a short time and then is vibrated manually by hitting it several times on a table corner. A short time after the preparation, when the liquid-air interface at the top of sample reaches the first layer of beads as the result of evaporation, the sample is placed on a Mettler-Toledo PB 3002-S precision scale with an accuracy of $0.01 \mathrm{~g}$ to measure solution mass loss at $2 \mathrm{~min}$ intervals with data automatically stored on a computer. As sketched in Fig. 1, the sample is set in a chamber of controlled relative humidity (relative humidity $\left.R H_{\infty} \approx 3 \pm 2 \%\right)$ and temperature $\left(T \approx 30^{\circ} \mathrm{C}\right)$. The temperature of $30^{\circ} \mathrm{C}$ is imposed using a thermo cryostat whereas the relative humidity is imposed thanks to a layer of dry silica gel particles spread out on the floor of the chamber. The relative humidity and temperature are recorded during the experiment using a KimoVT300 sensor set in the chamber and no noticeable evolution is noted during an experiment. A Nikon D100 camera with a resolution of $3008 \times 2000$ pixels is set above the sample. Top images of the efflorescence developing on sample surface are recorded with a frequency of 1 image every five minutes using the acquisition software Nikon Capture Control (version 3). A Elinchrom 300s flash system connected to the PC is used for the lighting so as to get images of good quality. The $\mathrm{NaCl}$ aqueous solution is prepared by adding a certain mass $m_{\text {salt }}$ of salt to a given volume $V$ of distilled water. To obtain a desired dissolved salt initial mass fraction $C_{0}$ ( $\mathrm{g}$ of NaCl$/ \mathrm{g}$ of solution), the mass $m_{\text {salt }}$ can be computed from the formula $m_{\text {salt }}=C_{0} V_{\frac{\rho(0)}{\left(1-0.7 C_{0}\right)}}$ where $\rho_{\ell}(0)$ is the density of pure water (the factor 0.7 in the denominator comes from a fit of the variation of solution density with salt mass fraction).

\section{Results}

\subsection{Drying regime for pure water}

The evolution of evaporated mass as a function of time for the various ranges of bead size for pure water is depicted in Fig. 2 along with results for $\mathrm{NaCl}$ aqueous solutions that will be discussed later.
The drying of capillary porous media is classically described in three main periods, e.g. [18] and references therein. During the first period, referred to as the CRP (constant rate period), the evaporation rate is essentially constant and controlled by the external demand (velocity and relative humidity in surrounding air). In this period, the surface is hydraulically well connected to the liquid contained in the porous medium, so that there is no limitation of evaporation due to transport phenomena taking place in the porous medium. The last period, the receding front period (RFP), is characterized by an internal evaporation front receding into the porous medium whereas the intermediate period, the falling rate period (FRP), is a crossover period characterized by a significant drop in the drying rate.

As can be seen from Fig. 2, our results with pure water are consistent with this classical description. However, the FRP seems to be very short and drying for pure water is in fact here characterized by essentially two main periods, a long quasi-constant rate period, during which most of the water contained in the porous medium evaporates, followed by a rather short falling rate period. There are some variations depending on the range of bead size but it can be observed that the evolution of evaporated mass is essentially the same for all the ranges of bead size with pure water. This drying regime with a long constant rate period is characterized by internal liquid distributions dominated by the capillary effects. As discussed in some details in $[19,20]$, the liquid saturation within the porous material decreases of course during the drying process but is expected to be spatially uniform during the constant rate period in this capillarity dominated regime.

\subsection{Efflorescence at the top surface}

In the presence of dissolved salt, the evaporation process is characterized by the development of efflorescence at the surface of the porous medium for all the salt concentrations and all the ranges of bead sizes considered in our experiments. This is illustrated in Fig. 3, which shows two examples of the efflorescence at the end of experiment. From previous works, i.e. [6,8], the formation of efflorescence is expected when the convective effect induced by the evaporation process is sufficient to induce greater salt concentration at the surface. The competition between the advection

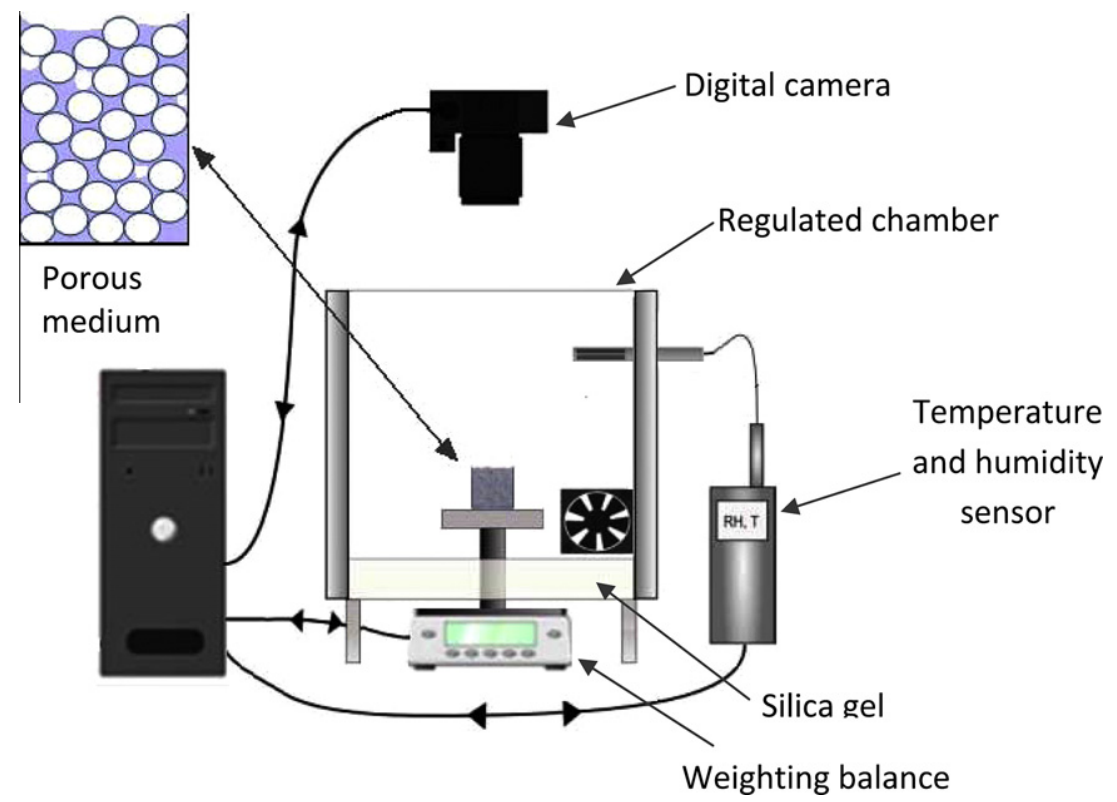

Fig. 1. Sketch of experimental set-up. The light source and the cryostat are not shown. 

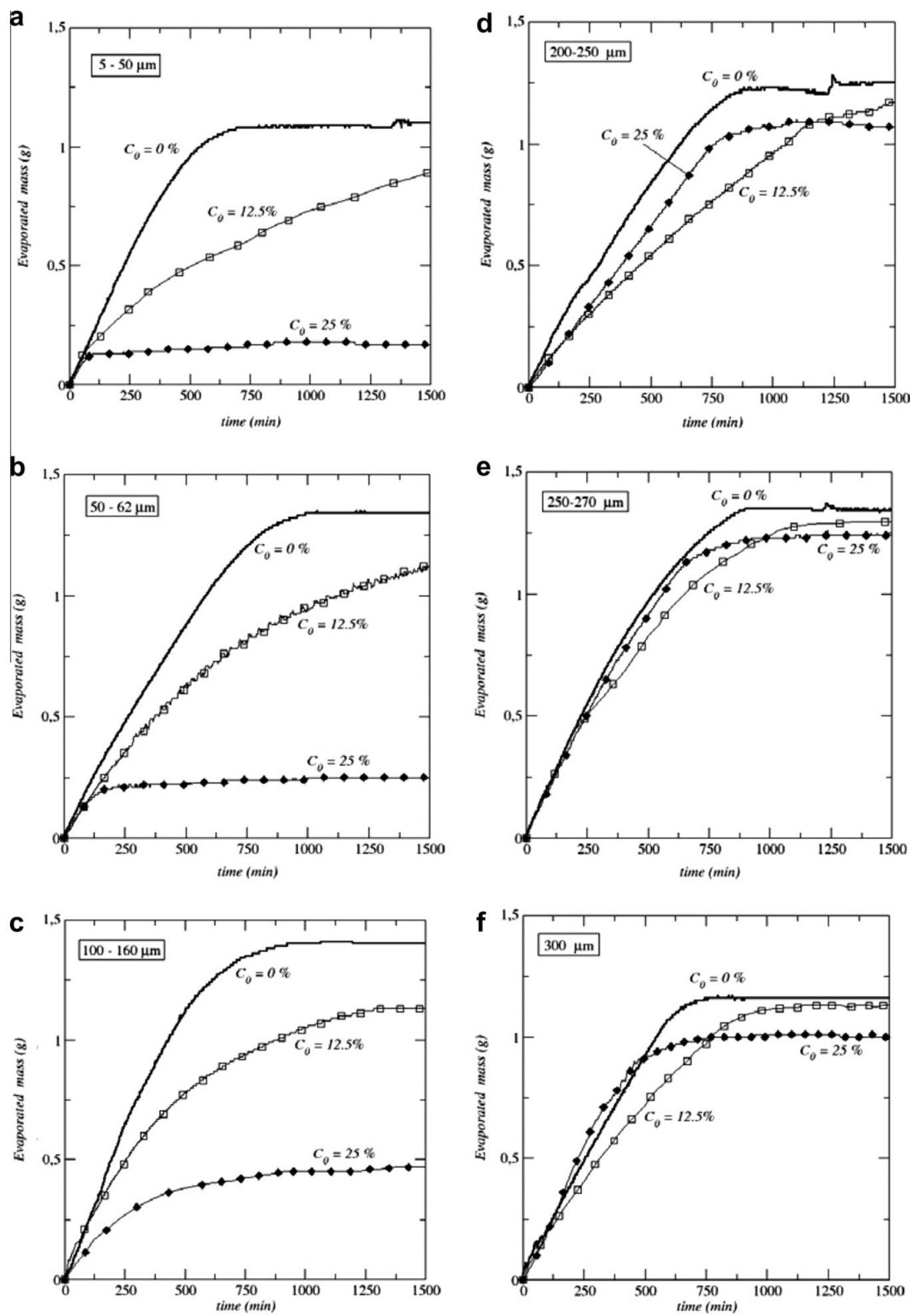

Fig. 2. Evolution of evaporated mass as a function of time for the various ranges of bead size and three different initial salt mass fractions. The efflorescence is crusty for the figures in the column on the left. The efflorescence is patchy for the figures in the column on the right.

effect, which tends to increase the salt concentration at the surface, and diffusion, which tends to make the concentration uniform across the sample, is usually characterized by the Peclet number at time $t=0$ (see also Section 3.3 below).

$P e=\frac{U \xi}{D_{\mathrm{s}}^{*}}$ where $U, \xi$ and $D_{s}^{*}$ are a characteristic velocity of the liquid in the porous medium, a characteristic ion transport length and the salt effective diffusion coefficient, respectively. The Peclet number can be interpreted as the ratio of the diffusion time $t_{\mathrm{d}}=\xi^{2} / D_{\mathrm{s}}^{*}$ to the convective time $t_{\mathrm{c}}=\xi / U$. For a packing of particles, $D_{\mathrm{s}}^{*} \approx 0.66 D_{\mathrm{s}}$ [21] where $D_{\mathrm{s}}=1.3 \times 10^{-9} \mathrm{~m}^{2} / \mathrm{s}$ is the salt diffusion coefficient. As discussed in [12], this value of $D_{\mathrm{s}}^{*}$ represents an upper bound 
a

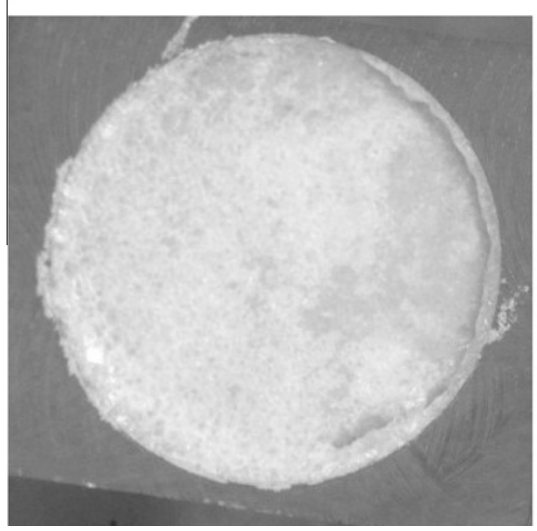

b

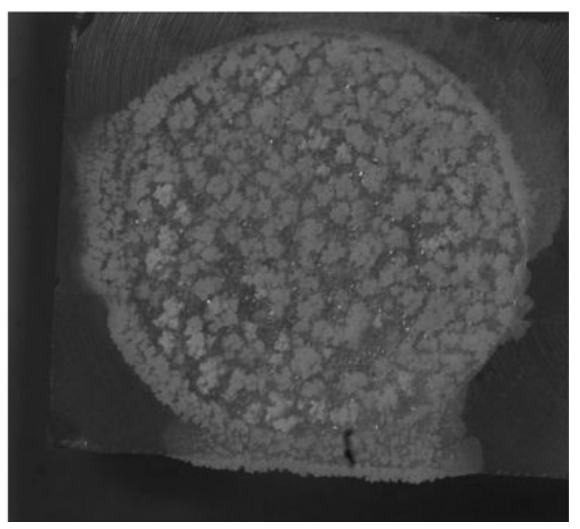

Fig. 3. Examples of efflorescence at the end of drying process for $C_{0}=25 \%$ : (a) range \#1 5-50 $\mu \mathrm{m}$, (b) range \#6 $300 \mu \mathrm{m}$. For the ranges of smaller glass beads from ranges \#1\#3, the efflorescence progressively forms a compact crust covering all the surface of porous medium at the end of drying. By contrast the efflorescence forms patches with a significant fraction of the surface free of efflorescence for the larger bead sizes, corresponding to the ranges \#4-\#6.

corresponding to a saturated sample, which is the situation at time $t=0$. In the case of a drying porous medium, this effective coefficient is expected to decrease with time since the liquid phase only occupies a fraction of the pore space. As explained in [6], an estimate of the velocity $U$ is given by $U=\frac{\bar{j}}{\varepsilon \rho_{\ell}}$ where $\bar{j}, \varepsilon$ and $\rho_{\ell}$ are the mean (= surface averaged) evaporation flux at the porous medium surface at time $t=0$, the porosity of the porous medium and the liquid density, respectively. From the results presented in the next sub-section, one can estimate an order of magnitude of the mean evaporation flux at the porous surface at the beginning of drying process. This gives $\bar{j} \approx 10^{-4} \mathrm{~kg} / \mathrm{m}^{2} / \mathrm{s}$, which in turn finally leads to $P e \approx 2.8$ (for $\varepsilon \approx 0.36$ and with $\xi=L$, where $L$ is the height of the sample which is the transport length classically considered in the definition of Peclet number in previous works, e.g. [6,7]). According to previous works, e.g. [6,7], a greater salt concentration at surface is expected when $P e>1$, that is when $t_{\mathrm{c}}<t_{\mathrm{d}}$.

Moreover it can be noted, as demonstrated in [14], that the flux is not uniform at the surface and greater at the periphery of the surface, at least at the beginning of the drying process. As noted in [14], the flux distribution is expected to be similar to the one obtained in the classical coffee ring problem in the limit of very small contact angle [22]. According to [23], this distribution can be expressed as,

$j(x) \approx j_{0}\left[1-\left(r / r_{\mathrm{c}}\right)^{2}\right]^{-1 / 2}$

where $r_{\mathrm{c}}$ is the radius of the cylinder, $r$ the radial distance from the middle of the porous surface and $j_{0}$ a prefactor, which is such that the integral of (2) over the surface should give the measured evaporation rate. This distribution is illustrated in Fig. 4. Note the divergence of the flux at the surface periphery. This indicates that the induced advection effect in the porous medium is in fact more marked than estimated from the average evaporation flux since the flux is not uniform over the surface and greater at the periphery. How this distribution is affected by the evolution of salt concentration at the surface and by the development of efflorescence is however not known and would deserve to be studied in a future work. As discussed in Section 3.8, the evolution of this distribution would notably depend on the salt initial mass fraction. Nevertheless, it can be safely assumed that the flux is non-uniform and greater at the periphery at the beginning of drying and for some time after the beginning, thus up to the efflorescence onset as discussed below.

\subsection{Efflorescence centripetal colonization}

The non-uniform flux distribution exemplified by Eq. (2) is confirmed in our experiments by the fact that the efflorescence first

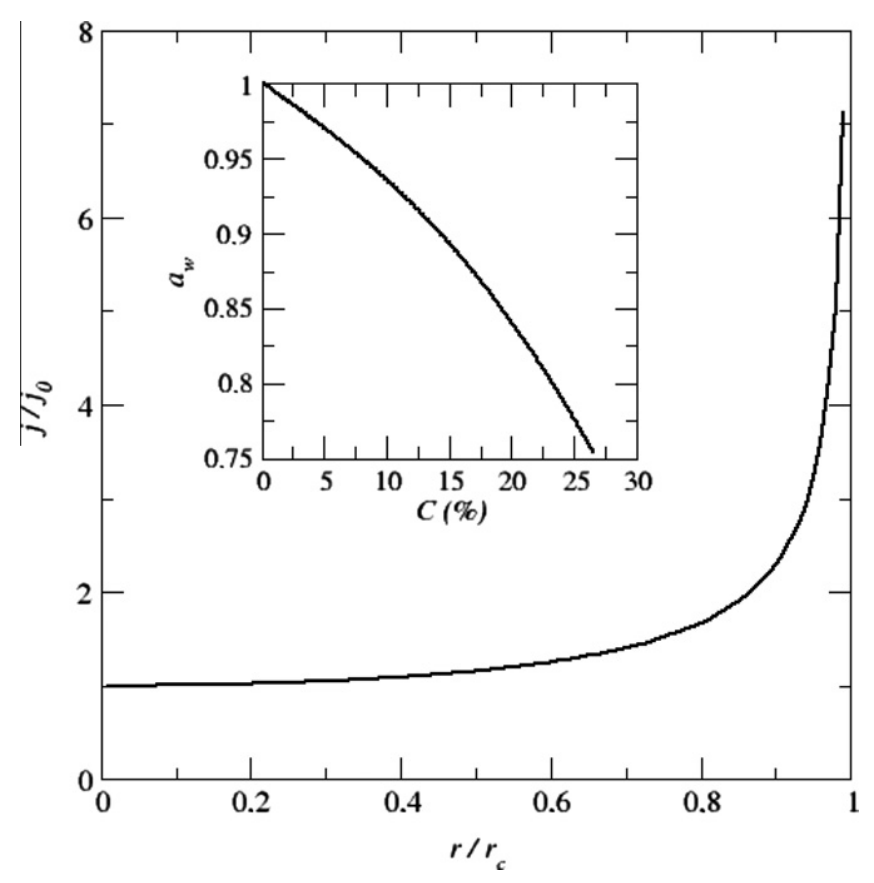

Fig. 4. Expected distribution of evaporation flux at the surface of the porous medium at the beginning of drying process. The evaporation flux is not uniform at the surface of porous medium and much greater at the surface periphery. The inset shows the evolution of water activity $a_{\mathrm{w}}=p_{\mathrm{v}}(\mathrm{C}) / p_{\mathrm{v} 0}$ as a function of dissolved salt mass fraction.

forms at the periphery of the surface. The onset of efflorescence at the periphery can be explained as follows. The dissolved salt mass transport within the sample is governed by the following equations,

$$
\begin{aligned}
& \frac{\partial \rho_{\ell} \varepsilon S C}{\partial t}+\nabla \cdot\left(\rho_{\ell} \varepsilon U S C\right)=\nabla \cdot\left(\rho_{\ell} S \varepsilon D_{\mathrm{s}}^{*} \nabla C\right) \\
& \left(\rho_{\ell} \varepsilon U S C-\rho_{\ell} S \varepsilon D_{\mathrm{s}}^{*} \nabla C\right) \cdot n=0
\end{aligned}
$$

where $U$ is the average interstitial velocity of the solution and $S$ the liquid saturation. The zero flux boundary condition (4), where $n$ is the unit vector normal to the considered surface, expresses that the dissolved salt cannot leave the porous medium before the onset of crystallization. 
At the porous medium surface during the period of rapid evaporation (see below), the evaporation flux is balanced at the surface of the porous medium by the liquid flow coming from the porous medium,

$$
\rho_{\ell} \varepsilon S U_{\mathrm{z}}=j
$$

where $U_{z}$ is the velocity component normal to the porous medium surface. It is clear from Eqs. (2) and (5) that the interstitial velocity in the region of the porous medium adjacent to the porous medium surface is greater at the periphery. As a result, the greater evaporation flux at the periphery induces a greater local instantaneous Peclet number at the periphery and therefore the preferential onset of the efflorescence at the periphery. This is sketched in Fig. 5. The local instantaneous Peclet number can be expressed as $P e=\frac{U_{z} \xi}{D_{s}^{\xi}}$, where the effective transport length $\xi$ at the periphery can be considered as slightly greater than the sample height $L$ because of the bending of streamlines toward the periphery within the porous medium. Thus, both the effect of a slightly longer transport length and a greater velocity contributes to the increase of the local instantaneous Peclet number at the periphery. As discussed in the next sub-section, the preferential evaporation at the periphery has also an impact on the efflorescence onset time.

As a result of the peripheral onset of efflorescence, the efflorescence then progressively invades the remaining of the surface from the porous medium surface periphery. This is shown in Fig. 6, which illustrates the centripetal colonization of surface by the efflorescence. This centripetal colonization process is observed for all the bead sizes and salt initial concentrations considered in our experiments.

\subsection{Efflorescence onset time}

The efflorescence onset time is estimated from the inspection of the images of the porous medium surface taken every $5 \mathrm{~min}$. The crystal should be therefore greater than about one pixel to be detected and there is an uncertainty of at most 5 min since the first crystal can appear between two images. The results are depicted in Fig. 7. These times are quite short compared to the period over which a significant evaporation exists (see Section 3.6). There is therefore little doubt that the efflorescence onset occurs during the first period of drying, that is when the surface is hydraulically well connected to the solution contained in the porous medium. An estimate of the efflorescence onset time was derived in [7] from the solution of the advection-diffusion equation, Eq. (3), governing the salt transport in the porous medium. This estimate reads,

$C_{\text {sat }}=C_{0}\left[1+\frac{P_{\mathrm{e}}^{2} \tau}{(1-P e \tau)^{2}}\right]$ where $\tau=t D_{\mathrm{s}}^{*} / L^{2}$ is the dimensionless crystallization time, using the diffusion time $t_{\mathrm{d}}$ with $\xi=L$ as reference time. As reported in Fig. 7, the results obtained from Eq. (3) with $P e=2.8$ leads to overestimate the efflorescence onset time. This is not truly surprising since several effects were neglected in the analysis developed in [7], namely the effect of the change in saturation on the effective coefficient $D_{s}^{*}$ as drying proceeds as well as the dependence of others properties like the solution viscosity, density or equilibrium vapor pressure with the salt concentration. Still more important is the fact, as noted before and illustrated in Fig. 4, that the evaporation flux is not uniform at the surface. This is in contrast with the assumption of uniform distribution considered in [7]. The smaller onset time observed in our experiments (except for 25\%) is consistent with the fact that the evaporation flux at the periphery of the surface is much greater than the average flux.

Another interesting aspect is the variation of onset time with the bead size. As illustrated in Fig. 7, it is found that the onset time does depend on the bead size, at least when the beads are small enough. There is clearly a difference between the bead size ranges \#4-\#6 and the smaller beads. Whereas the latter lead to an increase in the onset time as the mean bead size diminishes, this is not observed with the beads of the ranges \#4-\#6. This increase is not obvious to explain. From the divergence of the evaporation flux at the periphery (see Eq. (2)), one could have rather expected a decrease in the onset time for the smaller beads, since the finer the medium, the greater the evaporation rate from the menisci located at the periphery. The experimental results indicate exactly the opposite trend. These results are also at variance with the numerical simulations reported in [8], which indicate that the onset time should be independent of bead size as long as the efflorescence onset occurs in the first phase of drying, which is the case in our experiments as discussed later in the paper. As discussed in [8], the onset time is expected to be independent of bead size because the transport equation of dissolved salt (Eq. (3)) is independent of bead size (this is so because the effective diffusion coefficient in monodisperse bead packing is independent of bead size and because the induced velocity field and the saturation field in the sample during the first phase of drying are also independent of bead size for the capillarity dominated drying regime prevailing in our experiments (see [20] for more details). However, the simulations reported in [8] were for a uniform flux at the surface, which is not the situation in our experiments. One possible consequence of the greater flux at the periphery could be a faster drying at the periphery, that is lower saturations in the peripheral regions compared to the central region. This effect should preferentially occur with the smaller beads owing to the greater viscous resistance to the flow associated with the smaller beads (the permeability of the packing

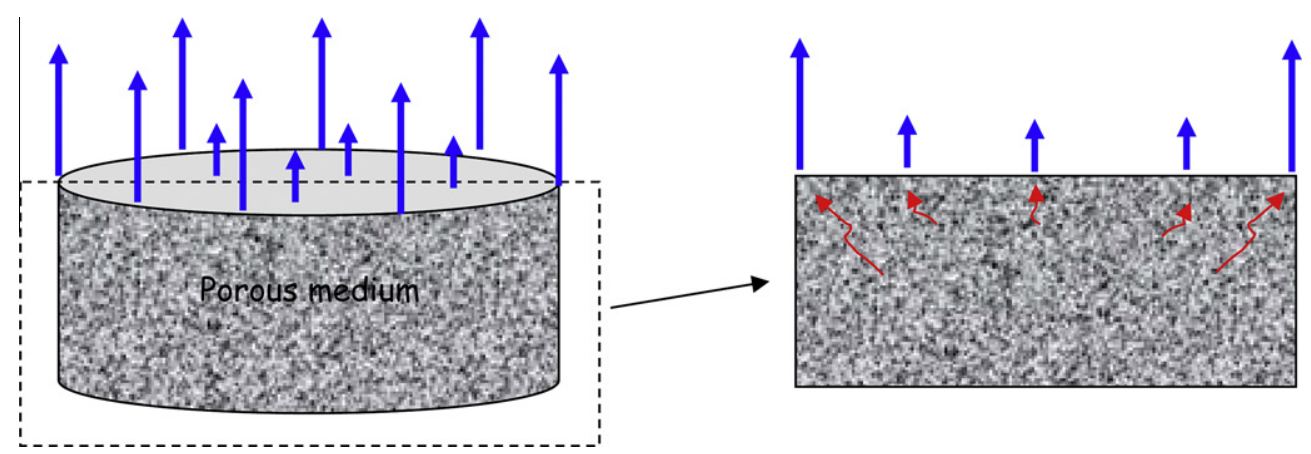

Fig. 5. Sketch of evaporation flux peripheral effect. The greater evaporation flux at the periphery induces greater liquid velocities within the region of the porous medium adjacent to the evaporative surface. These greater velocities induce in turn a greater transport by advection of the dissolved salt in the peripheral region, which leads to the preferential onset of efflorescence at the periphery. 
a

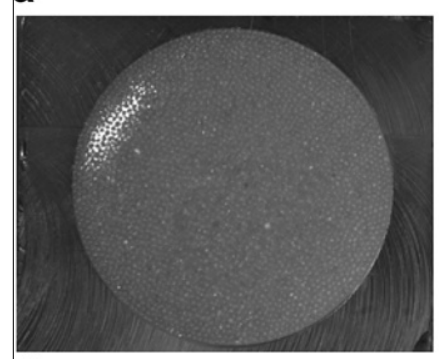

d

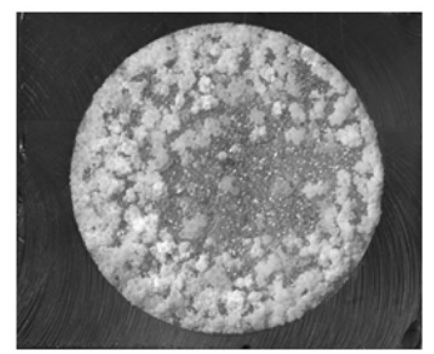

b

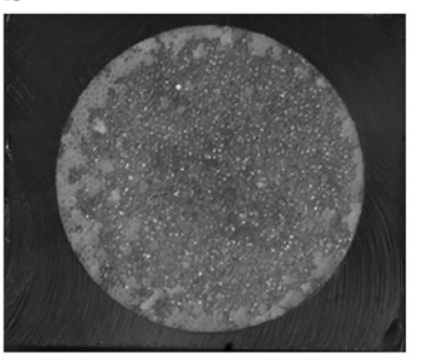

e

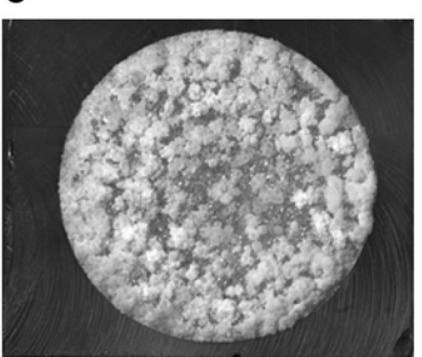

c
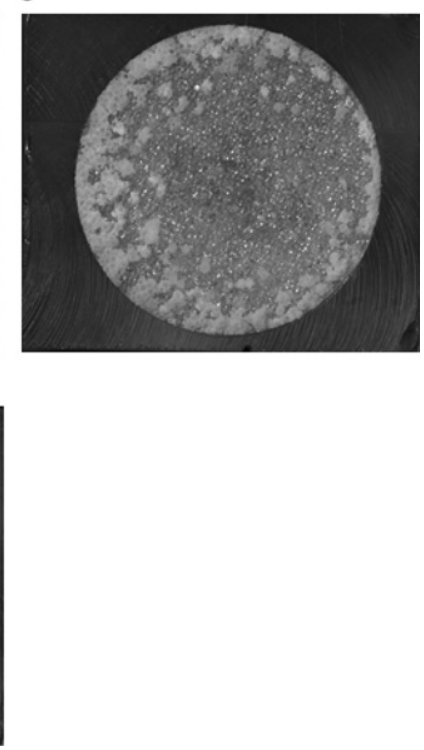

Fig. 6. Centripetal colonization of surface by the efflorescence. Top views of sample surface for the initial concentration $12.5 \%$ of NaCL for the packing made with beads of $300 \mu \mathrm{m}$ in diameter. (a) Top view at $t=0 \mathrm{~s}$, (b) top view at $t=2 \mathrm{~h} 41 \mathrm{~min}$, (c) top view at $t=3 \mathrm{~h} 51 \mathrm{~min}$, (d) top view at $t=7 \mathrm{~h} 1 \mathrm{~min}$, and (e) top view at $t=9 \mathrm{~h} 16 \mathrm{~min}$.

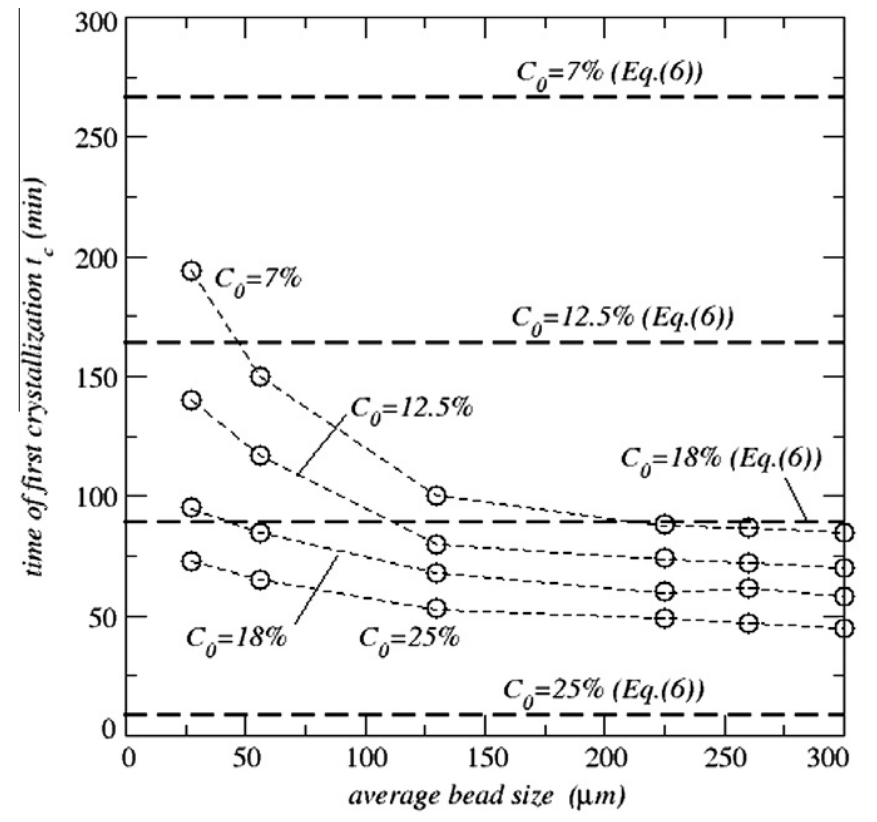

Fig. 7. Time of first crystallization as a function of average bead size for various initial concentrations (each empty circle corresponds to an experiment). The horizontal thick dashed lines corresponds to the theoretical prediction discussed in the text (Eq. (6)).

decreases with the bead size). This could perhaps explain the somewhat greater onset time observed in the experiments with the smaller beads.

However, the problem might also come from the images. If one assumes that an image contains about $2000 \times 2000$ pixels, this leads to a spatial resolution of about $100 \mu \mathrm{m}^{2} /$ pixel. Consider for instance the bead sizes in the range 50-62 $\mu \mathrm{m}$. According to [24], the mean pore size in a random packing of (monodisperse) particles is about one fourth of the bead size, that is here $\approx 14 \mu \mathrm{m}$, which gives a pore cross-section of about $150 \mu \mathrm{m}^{2}$. This clearly indicates that the crystal should occupy more than one pore to be detected in the case of the smallest beads ( $5-50 \mu \mathrm{m}$, for which estimating a mean pore size is more difficult than for a narrower distribution). Thus, further investigation is certainly needed to evaluate more properly the time of first crystallization in the case of the smaller beads.

As a result, the main conclusions of this section can be summarized as follows. First, the estimates of onset time based on simplified 1D theory, e.g. [6,7] are likely to be poor when the evaporation flux distribution is not uniform at the surface. Second, the onset time in our experiments is very short compared to the period of significant evaporation (this will be made clear shortly when we will discuss the evaporated mass curve). This is first indication that the efflorescence development occurs when the efflorescence is hydraulically well connected to the solution contained in the porous medium.

\subsection{Crusty or patchy efflorescence}

As illustrated in Fig. 3, a remarkable feature lies in the structure of the efflorescence with roughly two main types of efflorescence. For the ranges of smaller glass beads from range \#1 to range \#3, the efflorescence progressively forms a seemingly compact crust covering all the surface of porous medium at the end of drying. By contrast the efflorescence forms patches, sometimes more beautifully referred to as "cauliflowers", with a significant fraction of the surface free of efflorescence for the larger bead sizes, corresponding to the ranges \#4-\#6. In what follows the first type is therefore referred to as "crusty" whereas the second is referred to as "patchy". Patches were also obtained in the evaporationwicking experiments reported in [14], where it is explained that the patches are due to salt concentration heterogeneities at the surface induced by the spatial fluctuations in the velocity field within the porous medium due to the disordered nature of porous structure. As also discussed in [14], the porous nature of efflorescence patches could explain why the salt crystals continue to growth in separated structures rather than forming a uniform coat. In drying, there is in addition the fact that the larger pores get dry first as a result of capillary effects [19]. This can also contribute to the onset of efflorescence at some specific locations at the surface rather than everywhere. A more challenging problem is to explain why efflorescence does not always form patches but a crust when 
the beads are sufficiently small. First insights into this point are provided in Section 4 through the consideration of a simple model of capillary rise in the efflorescence structures.

Before closing this section, it can be noted that the destructive inspection of samples at the end of each experiments did not show the formation of significant subflorescence. Thus it can be safely assumed that most of crystallized salt is found in the efflorescence.

Let us now look at the effect of efflorescence on drying.

\subsection{Crust/patch transition and its impact on water recovery rate by evaporation}

In this sub-section, the influence of bead size on drying in the presence of salt is explored. Drying experiments for the various ranges of bead sizes were performed for three initial salt mass fractions, namely $0 \%$ (pure water), $12.5 \%$ and $25 \%$. It can be noted that the mass fraction $25 \%$ is close to the saturation mass fraction $C_{\text {sat }}$, which is $26.6 \%$ for $\mathrm{NaCl}$ at $30^{\circ} \mathrm{C}$. The evolution of evaporated mass as a function of time for the various ranges of bead size and the three salt mass fractions is depicted in Fig. 2.

Let us first consider the case of the highest initial salt initial mass fraction $\left(C_{0}=25 \%\right)$. In contrast with pure water, the evaporated mass curve for the initial concentration $25 \%$ strongly depends on bead size. One can distinguish two main situations. The first situation corresponds to the three smaller bead sizes, namely the ranges \#1-\#3. As can be seen from Fig. 2a-c, the evaporation rate becomes rapidly very small for these ranges and the total mass loss when the experiment is stopped is much smaller than for pure water. As shown in Fig. $2 \mathrm{~d}-\mathrm{f}$, the second situation, which is observed with the three larger bead sizes, is completely different with evaporated mass curves much more similar to the pure water curves. In contrast with the first situation, the results depicted in Fig. $2 d-f$ suggest that all the liquid water present initially has evaporated at the end of experiment. This can be confirmed by estimating the mass of liquid water present initially in the system. This mass is given by $m_{\mathrm{w} 0}\left(C_{0}\right)=\left(1-C_{0}\right) \rho_{\ell}\left(C_{0}\right) V_{\mathrm{p}}$, where $V_{\mathrm{p}}$ is the volume of the pore space within the porous medium. $V_{\mathrm{p}}$ can be estimated from the total mass of liquid evaporated when the liquid is pure water: $V_{\mathrm{p}}=m_{\mathrm{w} 0}(0) / \rho_{\ell}(0)$. This gives

$m_{\mathrm{w} 0}\left(C_{0}\right)=\left(1-C_{0}\right) \rho_{\ell}\left(C_{0}\right) m_{\mathrm{w} 0}(0) / \rho_{\ell}(0)$

Then we can define the fraction of water evaporated from the sample at the end of drying as $F=m_{\mathrm{w}}\left(C_{0}\right) / m_{\mathrm{wo}}\left(C_{0}\right)$, where $m_{\mathrm{w}}\left(C_{0}\right)$ is the total mass of water evaporated at the end of drying. The results are reported in Fig. 8. In the following, the factor $F$ is called the water recovery rate, or simply the recovery rate. As can be seen the presence of salt does not prevent evaporating about all the water in the samples when the bead size are sufficiently large whereas the "evaporation recovery" of water is poor when the average bead size is sufficiently small. Although the number of data is limited, one can clearly distinguish two main regimes in Fig. 8. A first regime corresponding to the first situation, where the recovery rate $F$ increases with the average bead size and a second regime, corresponding to the second situation, where the recovery rate is close to $100 \%$ independently of the bead size. Under our experimental conditions, the first regime is observed for the average bead size lower than $200 \mu \mathrm{m}$, whereas the second regime is observed for the average bead size $200 \mu \mathrm{m}$ and above. It would be interesting to perform experiment for smaller and smaller beads to see if the recovery rate becomes very small or reach a plateau before reaching an extremely small recovery rate.

Combined with the visual observation of efflorescence, which leads to distinguish the crusty efflorescence from the patchy efflorescence (Fig. 3), the above results indicate that the transition from crusty to patchy has a great impact on drying. As shown in Fig. 2ac, the crusty efflorescence tends to block (or severely limit) drying

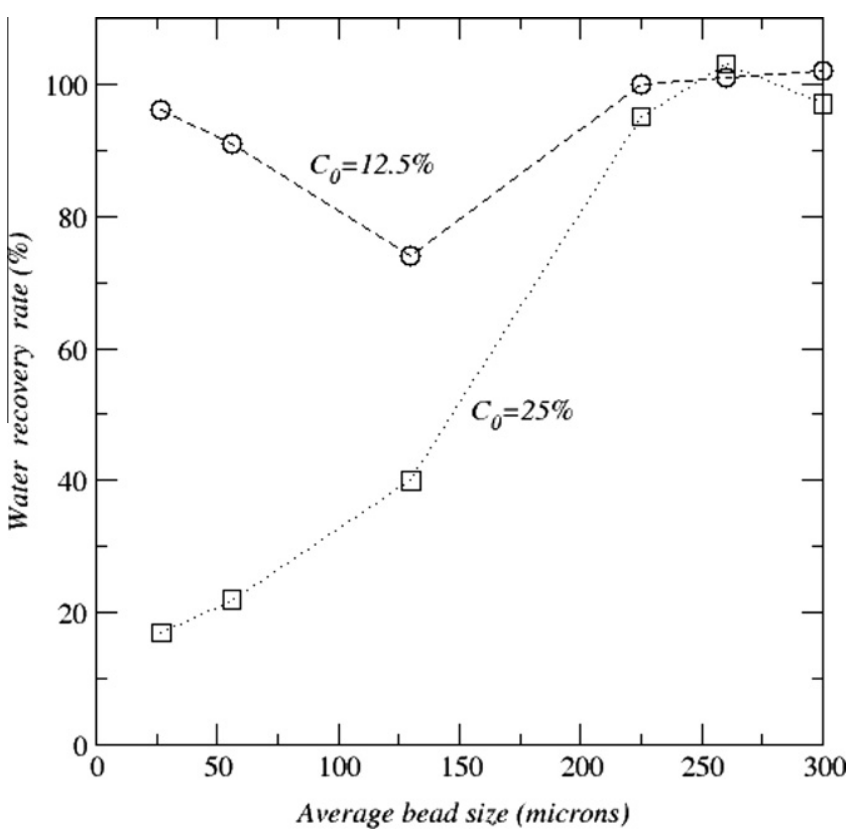

Fig. 8. Evolution of the fraction of initial mass of water evaporated at the end of drying as a function of mean bead size.

whereas, as shown in Fig. 2d-f, the patchy efflorescence leads to high drying rates in the first phase of drying, comparable to those observed with pure water. Interestingly, it can be seen from Fig. $2 f$ that the drying rate can be even greater than the drying rate for pure water and this is discussed in the next sub-section.

As illustrated in Fig. 2a-f. and in Fig. 8, the results are markedly different with the initial concentration $12.5 \%$ for the bead sizes lower than $200 \mu \mathrm{m}$. The water recovery is much higher in this range of bead sizes. One can also note a non-monotonous variation with the lowest water recovery rate obtained for the average bead size $130 \mu \mathrm{m}$. Further data will be desirable to confirm this nonmonotonous variation. The visual inspection of the efflorescence at the end of drying again indicates patchy efflorescence for the bead size ranges \#4-\#6 and crusty ones for the smaller bead sizes (ranges \#1-\#3). Thus, somewhat surprisingly, the crusty/patchy transition has much less impact on the water recovery than for the mass fraction $25 \%$.

Nevertheless, Fig. 2a-f, show an evolution of the evaporated mass curves with the bead size. The curves for $C_{0}=12.5 \%$ are again much more similar to that of pure water when the efflorescence is patchy. The drying rate is smaller in the first phase of drying (probably due to the impact of salt on the water activity) but the general shape of the curves is similar to the one for pure water. By contrast, the shape of the curves for this salt mass fraction is quite different when the efflorescence is crusty. For the smallest beads (range \#1), there is no first period anymore resembling to a constant rate period. The drying rate decreases progressively all along the drying process. This corresponds to the parabolic-like shape in Fig. 2a. This shape varies with the bead size (as depicted in Fig. $2 b-c$ ) and progressively tends toward the shape observed with the patchy efflorescence. Thus, although the type of efflorescence does not affect a lot the water total recovery rate, in contrast with the case $C_{0}=25 \%$, the fact that the efflorescence is patchy or crusty does have an influence and this is visible from the evaporated mass curves. This is further illustrated by the results discussed in what follows.

\subsection{Enhanced drying rates due to patchy efflorescence}

As noted before, it can be seen from Fig. $2 \mathrm{f}$ that the drying rate with the $300 \mu \mathrm{m}$ beads and the initial mass fraction $25 \%$ can be 
even greater than the drying rate for pure water. This result is completely counter-intuitive since the water activity $a_{\mathrm{w}}\left(a_{\mathrm{w}}=p_{\mathrm{v}}(C) / p_{\mathrm{v} 0}\right.$, where $p_{\mathrm{v}}(C)$ and $p_{\mathrm{v} 0}$ are the vapor pressure for a $\mathrm{NaCl}$ aqueous solution and the vapor pressure for pure water, respectively), is reduced in the presence of salts [25]. As depicted in the inset in Fig. 4, the water vapor pressure at the surface of an aqueous solution is a decreasing function of salt mass fraction. For the salt mass fraction of $25 \%$, the vapor pressure is about $75 \%$ of the vapor pressure for pure water. In our drying experiments, evaporation is driven by mass transfer. Thus the evaporation rate $J$ is proportional to $\left(p_{\mathrm{v}}(C)-p_{\mathrm{v} \infty}\right)$ where $p_{\mathrm{v}}(C)$ and $p_{\mathrm{v}_{\infty}}$ are the equilibrium vapor pressure at the surface of a liquid/gas interface and the vapor pressure in the regulated chamber, respectively. Thus, compared to pure water, the evaporation rate in the first phase of drying is expected

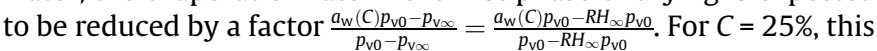
gives $J(C) \mid J(0) \approx 74 \%$, thus a reduction by a factor $25 \%$ compared to pure water. However, as mentioned before, the evaporation rate for $C=25 \%$ in Fig. $2 \mathrm{f}$ in the first phase of drying is not reduced but on the contrary enhanced compared to pure water. A similar observation was reported in [12] from experiments with model porous media obtained by sandwiching a monolayer of glass beads between two glass plates. The explanation was that the efflorescence is porous, pumps by capillarity the aqueous solution, and forms an enhanced exchange surface between the porous medium and the surrounding air. The enhanced exchange surface associated with the efflorescence thus overcompensates the reduction in the equilibrium vapor pressure due to the presence of salt. We believe that basically the same phenomenon occurs when the bead size is sufficiently large. Note that the effect can be due in part to the creeping (see [26]) of the salt along the walls adjacent to the porous sample surface. The creeping, which is visible in Fig. $3 \mathrm{~b}$ for example, also increases the exchange surface between the system and the surrounding air. Creeping around the sample surface was observed for the experiment with the range of bead size \#6 for which a drying rate greater than with pure water is observed.

\subsection{Influence of initial salt concentration on drying with patchy efflorescence}

In this subsection, we look at the effect of salt initial concentration on drying for the largest size of beads (range \#6, $300 \mu \mathrm{m}$ ), that is when the efflorescence is patchy. The evaporated mass curves for the various initial concentrations tested are depicted in Fig. 9. One can again distinguish a first phase where the evaporation is rapid followed by a short second phase where the evaporation rate decreases rapidly up to zero. The evaporation rate in the first phase (expressed in $\mathrm{g} / \mathrm{min}$ ) is estimated from the evaporated mass after time $t=t_{400}=400 \mathrm{~min}$, i.e. $J\left(C_{0}\right) \approx \frac{m_{400}}{t_{00}}$. The results are depicted in Fig. 10. As can be seen, the evaporation rate varies non-monotonously with the initial mass fraction with a minimum rate observed for the initial mass fraction $C_{0}=18 \%$. This could be already anticipated from Fig. 2f, which shows that the evaporation is slower in this phase for $C_{0}=12.5 \%$, compared to the cases $0 \%$ and $25 \%$. A naïve view based again on the effect on salt on the equilibrium vapor pressure would indicate that the dimensionless evaporation rate in this phase should scale as

$\frac{J\left(C_{0}\right)}{J(0)} \propto \frac{P_{\mathrm{v}}(C)-P_{\mathrm{v} \infty}}{P_{\mathrm{v} 0}-P_{\mathrm{v} \infty}}$

which, according to the inset in Fig. 4, would lead to a monotonous decreasing of evaporation rate with increasing initial salt concentration.

As discussed in Section 3.7, the fact that the evaporation rate is greater than for pure water for the initial mass fraction $25 \%$ can be explained by the enhanced exchange surface due to efflorescence.
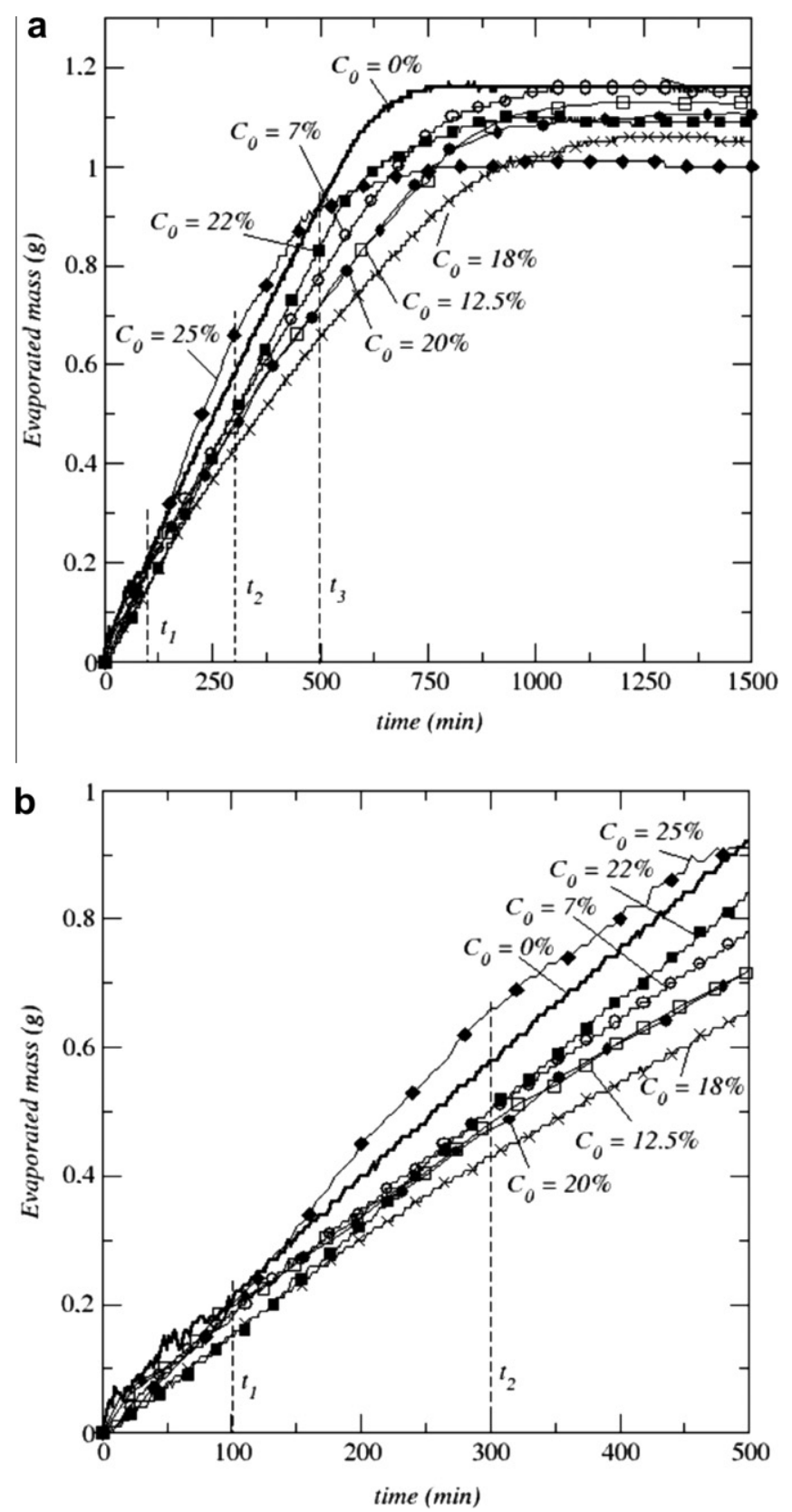

Fig. 9. Evolution of evaporated mass as a function of time for various salt initia mass fraction for the beads $300 \mu \mathrm{m}$ in diameter. Fig. 9a shows a detailed view of first $500 \mathrm{~min} . C_{0}=0 \%$ (thick solid line), $C_{0}=7 \%$ (thin solid line with empty circles) $C_{0}=12.5 \%$ (thin solid line with empty squares), $C_{0}=18 \%$ (thin solid line with crosses), $C_{0}=20 \%$ (thin solid line with solid circles), $C_{0}=22 \%$ (thin solid line with solid squares), $C_{0}=25 \%$ (thin solid line with solid diamonds).

The enhanced drying rate effect is a strong indication that the transfers occurring in the efflorescence play a very significant role in the non-monotonous behavior reported in Fig. 10. In brief, it is surmised that the non-monotonous behavior with the initial salt concentration in Fig. 10 is a consequence of the coupling between the evaporation process and the transfers in both the porous medium and the growing efflorescence. It can be noted that such a nonmonotonous behavior was also observed in the experiments with a micromodel presented in [13] as well as in the drying experiments from long sand column discussed in [16]. As in our experiments, these experiments are characterized by the major development of efflorescence in the first phase of drying. 


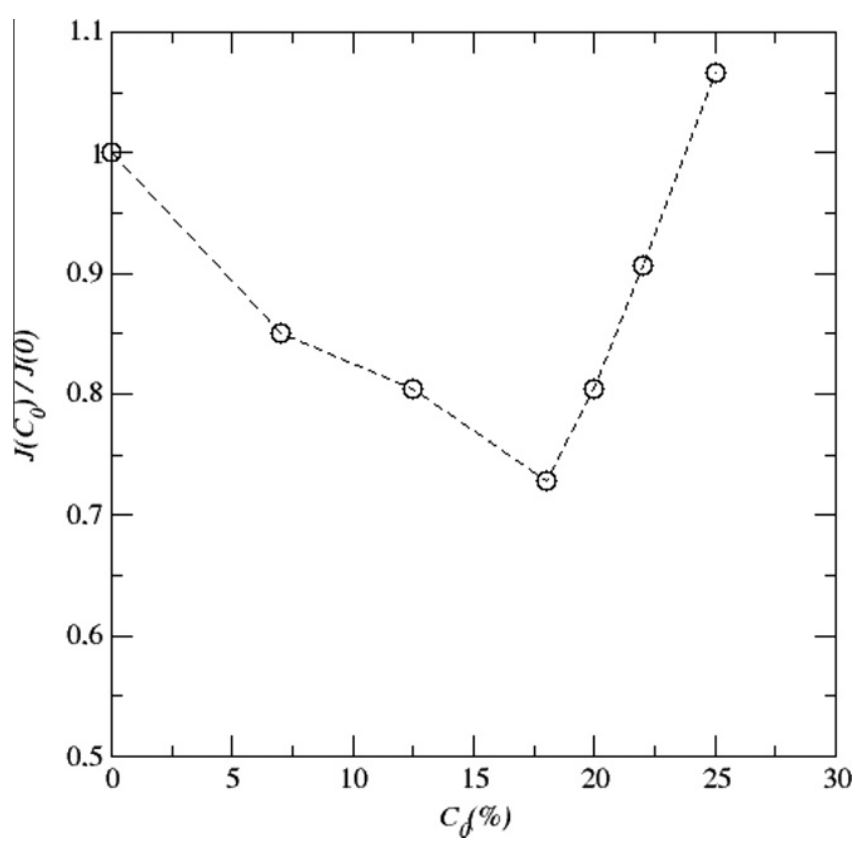

Fig. 10. Evolution of evaporation rate as a function of salt initial mass fraction in the first phase of drying (see text) for the beads of $300 \mu \mathrm{m}$ in diameter.

The understanding of this coupling is of course desirable but more work is needed to develop a detailed analysis. One can gain, however, some first insights from the top views of efflorescence presented in Fig. 11. First, one can note that the efflorescence covers practically all the surface at $t=300 \mathrm{~min}$ for the salt initial mass fraction in the range [18\%, 25\%], which corresponds to the increasing branch of the curve in Fig. 10. Second, the efflorescence is clearly more developed at $t=300 \mathrm{~min}$ as the salt initial mass fraction increases in this range of salt initial mass fraction [18\%, 25\%]. Thus here one can conclude that the increase in the drying rate with the salt initial mass fraction after the minimum drying rate in Fig. 10 (which corresponds to $C_{0}=18 \%$ in Fig. 10), is simply due to the increase in the exchange surface associated with the efflorescence. This argument does not hold, however, for the decreasing branch of the curve in Fig. 10. From Fig. 11, it is clear than the efflorescence is more developed as the salt initial mass fraction increases also in the range of salt initial mass fractions ] $0 \%, 18 \%]$, whereas the drying rate decreases. In contrast with the case of the higher salt initial mass fractions, one can note however that a large fraction of the porous medium surface is free of efflorescence at $t=300 \mathrm{~min}$ for $C_{0}=7 \%$ and $12.5 \%$. The absence of efflorescence in the central region is an indication that the salt concentration at the surface in the central region free of efflorescence is less than the saturation mass fraction $C_{\text {sat }}$. As a result, the vapor pressure is greater in the central region than in the peripheral region where the efflorescence is present since as discussed before and shown in the inset in Fig. 4, the vapor pressure is a decreasing function of the salt concentration. It follows that the evaporation flux should be greater in the central region free of efflorescence since the flux varies as $\left(p_{\mathrm{v}}(C)-p_{\mathrm{v}_{\infty}}\right)$. In passing this would be an indication that the flux distribution depicted in Fig. 4 is only valid at the beginning of drying, at least when the salt initial concentration is small enough owing to the evolution of salt concentration along the surface of porous medium. It is therefore surmised that the salt concentration at $t=300 \mathrm{~min}$ in the central region is lower for $C_{0}=7 \%$ than for $C_{0}=12.5 \%$. (this is confirmed by the top views of efflorescence at $t=500 \mathrm{~min}$, which shows a greater surface free of efflorescence for the case $C_{0}=7 \%$ ), which therefore would lead to a greater overall evaporation rate for the case $7 \%$ compared to the case $12.5 \%$.
In summary, for a sufficiently high salt mass fraction, the salt concentration distribution is rapidly more uniform and close to the saturation concentration, which leads to the rapid development of efflorescence over the entire surface. The dominant effect, which explains the increasing branch in Fig. 10, is then the increase in the efflorescence exchange surface with the salt initial mass fraction. For a sufficiently low salt initial mass fraction, a non-uniform salt concentration distribution develops at the surface with lower concentrations, and thus greater evaporation fluxes, in the central region over a significant period of drying. This non-uniform distribution effect would be more marked as the initial concentration decreases, which explains the decreasing branch of the curve in Fig. 10. Thus the decreasing branch in Fig. 10 is explained by the dominant effect of the variation of water activity with salt concentration whereas the ascending branch is explained by the evaporation enhancement effect due to major efflorescence developments.

Before closing this section, two points can be noted. First, a similar non-monotonous behavior is expected for the bead size ranges \#4 and \#5 since again the results depicted in Fig. $2 \mathrm{~d}$ and e shows that evaporation is slower in the first phase for $C_{0}=12.5 \%$, compared to the cases $0 \%$ and $25 \%$. Thus it is conjectured that such a non-monotonous behavior will be observed when the efflorescence is patchy.

\subsection{Influence of initial salt concentration on drying with crusty efflorescence}

By contrast, Fig. 2a-c indicates a different behavior when the efflorescence is crusty since the mean evaporation rate in the first phase decreases with increasing concentration. The variation of mean evaporation rate with initial concentration should therefore be monotonous in this case. This is confirmed by the results depicted in Fig. 12 for the bead size range \#2 (50-62 $\mu \mathrm{m})$. The efflorescence is crusty and clearly the mean evaporation flux in the phase of rapid evaporation decreases monotonously with the increasing initial salt concentration. This is also illustrated in Fig. 13, which shows the variation of the fraction of initial mass of water evaporated at the end of experiments.

In contrast with the patchy efflorescence, the crusty efflorescence blocks or severely limits the evaporation. Thus, evaporation in this case should therefore essentially take place from the fraction of the porous medium surface free of efflorescence. This efflorescence free evaporation surface area shrinks during drying as a result of the centripetal colonization of the surface by the efflorescence (see Section 3.3). The lower the area of the surface free of efflorescence, the lower the evaporation rate. Combined with the fact the water activity decreases as the salt concentration increases, this should explain the monotonous decrease of the mean evaporation flux with the increasing initial salt concentration when the efflorescence is crusty for the evaporative surface shrinking occurs faster for a greater initial salt concentration. A more complete analysis should also take into account the fact that the menisci at the surface first recede in the large pores at the surface as the result of drying. It is therefore possible that some pores at the interface are free of liquid and remains free of efflorescence, thus providing passages for the vapor between the internal menisci and the external air. This is more likely to happen with a low initial salt concentration. This could explain why a very high recovery rate of water is obtained for the lower initial salt concentrations in Fig. 13.

\section{A simple phenomenological model of efflorescence growth}

To explain the crusty-patchy transition observed in the experiments as the bead size is varied, we propose in this section a 


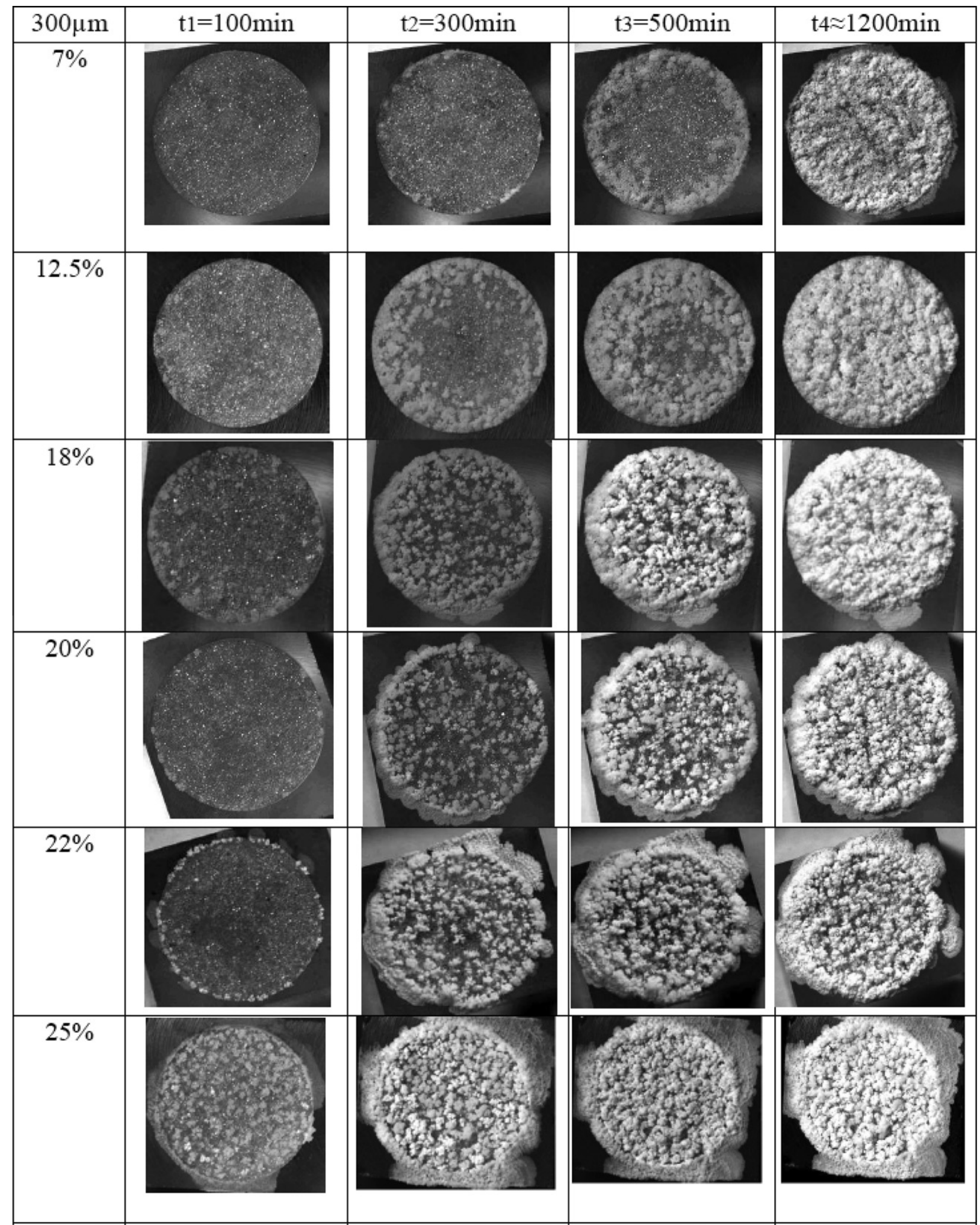

Fig. 11. Top view of sample surface at different times for the various initial concentrations for the packing made with beads of $300 \mu \mathrm{m}$ in diameter.

simple phenomenological model. It should be clear that the detailed understanding of this transition needs much more work owing to the complexity of the phenomena involved in the process but we believe that this simple model gives some interesting insights. The model is based on a series of rather crude assumptions and simplifications. First, we consider that the efflorescence is patchy at the very beginning independently of the bead size. This is what happens after this patchy phase which decides whether the efflorescence is going to remain patchy or become crusty. For simplicity the individual salt structures are modeled as porous 


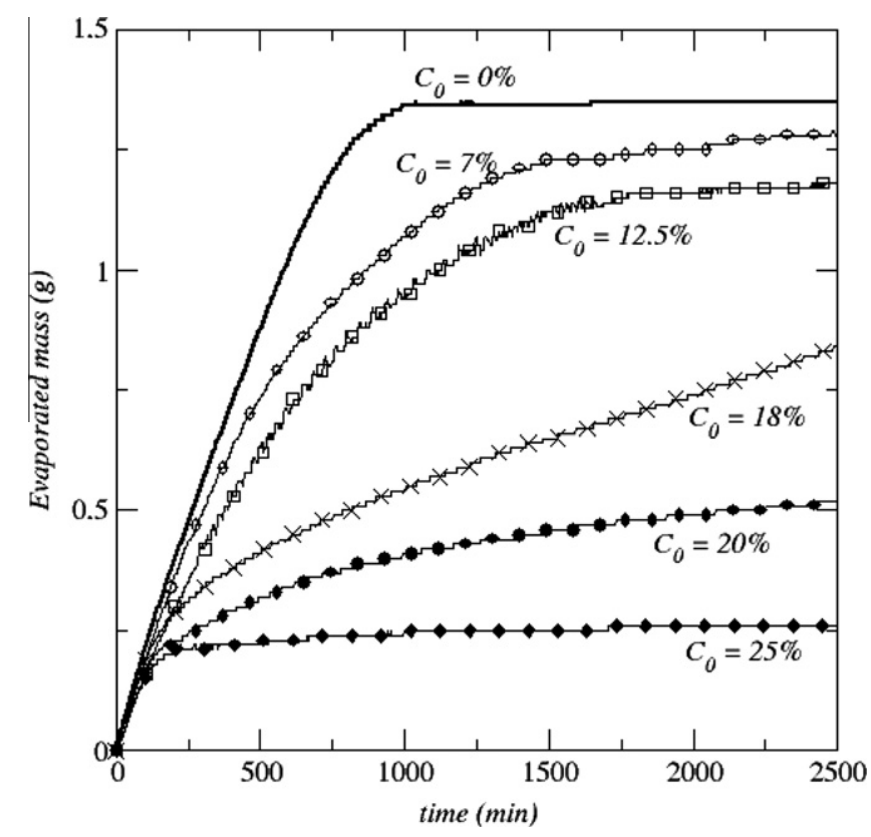

Fig. 12. Evolution of evaporated mass as a function of time for various salt initial mass fractions for the beads $50-62 \mu \mathrm{m}$ in diameter. $C_{0}=0 \%$ (thick solid line), $C_{0}=7 \%$ (thin solid line with empty circles), $C_{0}=12.5 \%$ (thin solid line with empty squares), $C_{0}=18 \%$ (thin solid line with crosses), $C_{0}=20 \%$ (thin solid line with solid circles), $C_{0}=22 \%$ (thin solid line with solid squares), $C_{0}=25 \%$ (thin solid line with solid diamonds).

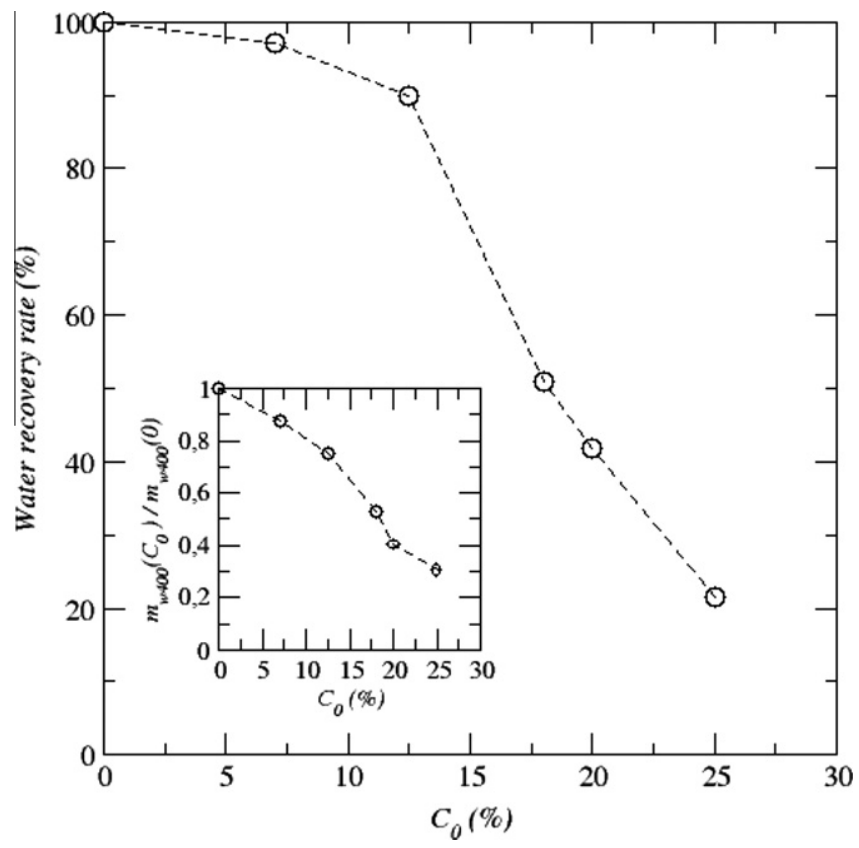

Fig. 13. Evolution of the fraction of initial mass of water evaporated at the end of experiment $(t=2500 \mathrm{~min})$ as a function of initial concentration for the beads 50 $62 \mu \mathrm{m}$ in diameter The inset shows the evolution of dimensionless evaporated mass at $t=400 \mathrm{~min}$ as a function of salt initial mass fraction for the beads $50-62 \mu \mathrm{m}$ in diameter.

cylinders of height $h_{\mathrm{e}}$ and diameter $d_{\mathrm{e}}=d$, where $d$ is the bead diameter. The mean distance $a$ between the patches is also proportional to the bead size. We took $a \approx 2 d$ as explained below. The porosity of salt cylinder is $\varepsilon_{\mathrm{e}}$ independently of bead size. The permeability of salt cylinder does depend on bead size. A simple model is to assume that the mean pore size in salt structures is linearly proportional to the mean pore size in the underlying porous medium. Assuming for simplicity that the salt structure is formed by a packing of grains of mean diameter $\delta_{\mathrm{e}}$ we thus assume that $\delta_{\mathrm{e}}=b d$, where $b$ is a numerical factor. It is assumed that the mean grain size of the salt structure is smaller than the mean bead size in the underlying porous medium and this will be taken into account through the choice of the value for $b$. Using the standard KozenyCarman relationship, the permeability of the salt structure is thus expressed as $K_{\mathrm{e}}=b^{2} \frac{\varepsilon_{\mathrm{e}}^{3} d^{2}}{\left(1-\mathrm{c}^{2}\right)^{2}}$. We suppose that each cylinder grows as a result of salt deposition at its top surface. Thus at the top surface of area $A=\pi d_{\mathrm{e}}^{2} / 4$, the mass flow rate $Q$ induces within the efflorescence structure should balance the evaporation rate,

$Q=A j_{\mathrm{e}}$

where $j_{\mathrm{e}}$ is the evaporation flux at the top of efflorescence. Owing to the screening of the surface of the porous medium free of efflorescence between the salt structures, it is assumed that the evaporation takes place essentially at the top of salt structures. One can refer to [14] for more details on the screening effect. Assuming that the salt structures of diameter $d$ form a square array and are at a distance $2 d$ of each other (this is illustrated in Fig. 14). The evaporation flux at the top surface of salt structures can be expressed as $j_{\mathrm{e}} \approx \frac{4 d^{2}}{\left(\pi d^{2} / 4\right)} j$, that is $j_{\mathrm{e}} \approx 5 j$.

Each salt cylinder is saturated by the solution owing to the capillary rise. To express the capillary pressure on top of the cylinder, we use an expression developed for packing of particles [27], $P_{\mathrm{ce}}=c \frac{\left(1-\varepsilon_{\mathrm{e}}\right) \sigma}{\varepsilon_{\mathrm{e}} \delta_{\mathrm{e}}}=\frac{c}{b} \frac{\left(1-\varepsilon_{\mathrm{e}}\right) \sigma}{\varepsilon_{\mathrm{e}} d}$, where $c$ is a numerical factor, $\sigma$ is the surface tension and where we have assumed that the solution was perfectly wetting. Then using Darcy'law, the flow rate in the saturated salt cylinder can be expressed as,

$Q=-A \rho_{\ell} \frac{K_{\mathrm{e}}}{\mu}\left(\frac{P_{\ell}\left(h_{\mathrm{e}}\right)-P_{\ell}(0)}{h_{\mathrm{e}}}+\rho_{\ell} g\right)$

where $\mu$ is the solution viscosity, $g$ the gravitational acceleration, $\rho_{\ell}$ is the solution density, $P_{\ell}\left(h_{\mathrm{e}}\right)$ is the pressure in the liquid at the top of the cylinder whereas $P_{\ell}(0)$ is the pressure in the liquid at the bottom of the cylinder. The latter can be expressed considering the capillary pressure jump at the menisci at the surface of the porous medium free of efflorescence. For a random packing of spherical particles, this capillary pressure can be expressed as

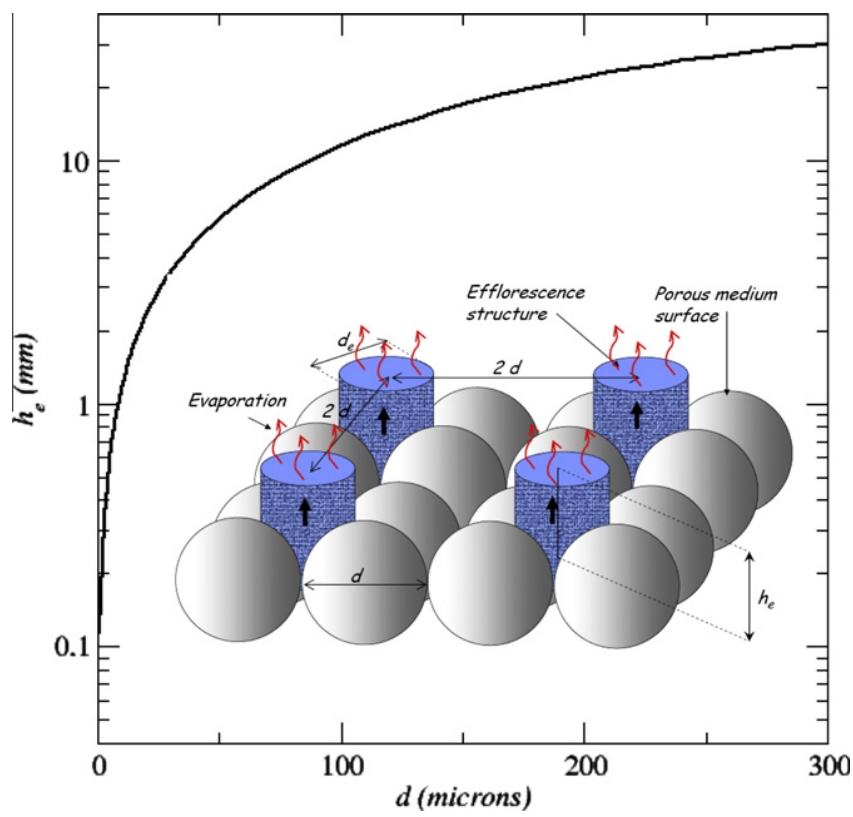

Fig. 14. Efflorescence structure height as a function of bead diameter (Eq. (12)). 
$P_{\mathrm{cpm}}=\frac{6(1-\varepsilon) \sigma}{\varepsilon d}=P_{\mathrm{atm}}-P_{\ell}(0)$ [27], where $\varepsilon$ is the porosity of the porous medium. At the top of the cylinder, we have a similar expression, $P_{\text {ce }}=\frac{c}{b} \frac{\left(1-\varepsilon_{\mathrm{e}}\right) \sigma}{\varepsilon_{\mathrm{e}} d}=P_{\mathrm{atm}}-P_{\ell}\left(h_{\mathrm{e}}\right)$. Combining the above equations leads to

$j_{\mathrm{e}}=-\rho_{\ell} \frac{K_{\mathrm{e}}}{\mu}\left(\frac{P_{\mathrm{cpm}}-P_{\mathrm{ce}}}{h_{\mathrm{e}}}+\rho_{\ell} g\right)$

from which one can obtain an estimate of the maximum height of each cylinder as,

$h_{\mathrm{e}}=\sigma d\left(\frac{\frac{c}{b} \frac{1-\varepsilon_{\mathrm{e}}}{\varepsilon_{\mathrm{e}}}-6 \frac{1-\varepsilon}{\varepsilon}}{\rho_{\ell} g d^{2}+j_{\mathrm{e}} \frac{v\left(1-\varepsilon_{\mathrm{e}}\right)^{2}}{b^{2} \varepsilon_{\mathrm{e}}^{3}}}\right)$

where $v$ is the kinematic viscosity of the solution. This equation can be expressed in dimensionless form as

$\frac{h_{\mathrm{e}}}{d}=\left(\frac{\frac{c}{b} \frac{1-\varepsilon_{\mathrm{e}}}{\varepsilon_{\mathrm{e}}}-6 \frac{1-\varepsilon}{\varepsilon}}{B+C a}\right)$

where $B=\frac{\rho_{\ell} g d^{2}}{\sigma}$ is the Bond number, which characterizes the competition between capillary and gravity effects in the salt structure, whereas $C a=\frac{j_{e} v\left(1-\varepsilon_{e}\right)^{2}}{b^{2} \varepsilon_{j}^{3} \sigma}$ is the capillary number, which characterizes the competition between capillary and viscous effects in the salt structure. Thus, Eq. (13) expresses that the efflorescence structures cannot exceed a certain height given by Eq. (13). This maximum height is directly related to the capillary rise in the salt structure. As for classical wicking problems in porous media [27] the rise can be limited by gravity effects, viscous effects or both.

The variation of the salt structures height $h_{\mathrm{e}}$ with the bead diameter has been computed using Eq. (12) with the following values of the parameters: $j \approx 10^{-4} \mathrm{~kg} / \mathrm{m}^{2} / \mathrm{s}$ (which is representative of our experiments), $\varepsilon_{\mathrm{e}}=0.04, \quad \varepsilon_{\mathrm{mp}}=0.36, \quad c=6 \times 10^{-2}, \quad b=10^{-2}$. (which corresponds to a mean pore size in the salt structure 100 times smaller than the mean pore size in the underlying packing of beads). The results are plotted in Fig. 14. As can be seen, the height for the larger beads is centimetric whereas the maximum height decreases sharply for the bead size lower than about $100 \mu \mathrm{m}$. For the size $26 \mu \mathrm{m}$ (range \#1), the maximum height in Fig. 14 is on the order of $2-3 \mathrm{~mm}$. These orders of magnitude are consistent with the experimental observations.

Nevertheless, we are not of course claiming that this simple model can lead to truly quantitative predictions since the values chosen for the parameters can be certainly questioned as long as the many assumptions. For example we have implicitly assumed the self-similarity of the salt structures with the bead size. The reality is probably more complex. Also, we have neglected the Kelvin's effect, i.e. the reduction of water activity due to high curvature of menisci in sufficiently small pores, whereas a somewhat more sophisticated version of the model for the simpler case of wicking-evaporation indicates that it is more consistent to take it into account [28]. The essential merit of this simple model is rather to suggest the following scenario. For sufficiently large bead sizes the efflorescence structures can grow upward up to a significant height and this leads to the patchy efflorescence. By contrast, the upward growth of salt structures would be rapidly limited owing to the combined action of gravity and viscous effects (and Kelvin's effect) when the bead size is sufficiently small. As the results, the incipient patches would grow laterally rather than vertically, coalescing rapidly with each other to form a crust.

This simple model also suggests that the patchy-crusty transition should also occur for a given bead size when the evaporation flux is sufficiently varied. In the case of our experiments, the efflorescence could become crusty with the larger bead sizes if the evaporation flux is sufficiently increased (by using a blower for instance or by increasing the temperature) or could become patchy with the smaller beads if the evaporation flux is sufficiently reduced (by increasing the relative humidity in the chamber for example). We have performed preliminary experiments that seem in agreement with these ideas but more work is needed to confirm these qualitative predictions.

\section{Conclusion}

Understanding and modeling the drying process of a porous medium in the presence of salt is an exciting challenge. As reported in this article, many questions are still widely open. We hope that the findings reported in the present article will stimulate further works. These findings can be summarized as follows:

(1) The presence of the salt can have a great impact on the drying of a porous medium.

(2) Two main types of efflorescence, referred to as crusty and patchy have been identified.

(3) The evaporated mass curves are quite different depending on whether the efflorescence is patchy or crusty.

(4) When the efflorescence is crusty, the crystal crust that forms at the surface of the porous medium can severely limit the evaporation. The blocking of evaporation due to the formation of a crystallized salt crust at the surface of the porous medium can be very effective with only a small fraction of the water initially present in the medium evaporating.

(5) When patches form the evaporation is not significantly limited by the presence of the crystallized salt at the surface of the porous medium. All the water initially present in the medium can evaporate. The evaporation rate can be in fact even greater than with pure water owing to the enhanced evaporation surface corresponding to the outer surface of salt structures.

(6) The two situations, referred to as patchy and crusty, can be observed for the same evaporation condition. The crusty efflorescence is observed for a sufficiently finer porous medium whereas the patchy efflorescence is observed for a sufficiently coarse porous medium. This has been observed in our experiments by varying the average size of the beads forming the porous medium.

(7) According to our experiments, the occurrence of a patchy (or a crusty) efflorescence does not depend on the initial concentration for a given bead size and for given evaporation condition; at least as long as a significant efflorescence develops at the surface (very low initial salt concentrations have not been tested).

(8) A simple qualitative model of efflorescence based on the assumption that the efflorescence behaves as a porous wick suggests that the crusty-patchy transition should be also observed for a given porous medium by varying the evaporation rate.

(9) When the efflorescence is patchy, the evaporation rate in the phase of rapid evaporation where the surface of the porous medium is hydraulically well connected to the solution present in the pore space is a non-monotonous function of the initial salt concentration. This is attributed to the complex interplay between the efflorescence development and the various transport phenomena taking place within the porous medium, the efflorescence structures and the gas phase.

(10) When the efflorescence is crusty, such a non-monotonous variation is not observed anymore. The mean evaporation rate decreases monotonously with the initial salt concentration. This is a consequence of the combined effect of the reduction in water activity with the salt concentration and the reduction of the porous medium surface available for 
evaporation since there is a blockage of the evaporation over the area of the surface of the porous medium covered by the efflorescence.

(11) The development of the efflorescence depends on the distribution of the evaporation flux at the surface of the porous medium. In the case of our experiments, the flux is greater at the surface periphery (at least at the beginning of the process) and this leads to a centripetal development of efflorescence. The efflorescence starts at the periphery and progressively develops toward the center of the surface of the porous medium.

(12) The efflorescence onset time is found to be independent of bead size, in agreement with a previous work, but only for the larger bead sizes considered. The influence of bead size on the onset time for the smaller beads needs to be clarified.

(13) The analytical estimate of onset time proposed in a previous work from the simplified solution of the salt transport equation overestimate the onset time. This is attributed to the numerous non-linearities neglected in the analytical approach and to the non-uniform evaporation flux distribution at the surface of the porous medium.

(14) All the results presented in this article were obtained with $\mathrm{NaCl}$ aqueous solutions. Considering another salt, or salt mixtures, could lead to different results and this would be worth studying.

\section{References}

[1] U. Nachshon, N. Weisbrod, M.I. Dragila, A. Grader, Combined evaporation and salt precipitation in homogeneous and heterogeneous porous media, Water Resources Research 47 (2011) W03513.

[2] Y. Peysson, B. Bazin, C. Magnier, E. Kohler, S. Youssef, Permeability alteration due to salt precipitation driven by drying in the context of $\mathrm{CO}_{2}$ injection, Energy Procedia 4 (2011) 4387-4394.

[3] A. Goudie, H. Viles, Salt Weathering Hazards, Wiley, Chichester, 1997.

[4] G.W. Scherer, Stress from crystallization of salt, Cement and Concrete Research 34 (2004) 1613-1624.

[5] C. Rodriguez-Navarro, E. Doehne, Salt weathering: influence of evaporation rate, supersaturation and crystallisation pattern, Earth Surface Processes and Landforms 24 (1999) 191-209.

[6] H.P. Huinink, L. Pel, M.A.J. Michels, How ions distribute in a drying porous medium: a simple model, Physics of Fluids 14 (4) (2002) 1389-1395.

[7] L. Guglielmini, A. Gontcharov, A.J. Aldykiewicz, H.A. Stone, Drying of salt solutions in porous materials: intermediate-time dynamics and efflorescence, Physics of Fluids 20 (2008) 077101.
[8] N. Sghaier, M. Prat, S. Ben Nasrallah, On ions transport during drying in a porous medium, Transport in Porous Media 62 (2007) 243-274.

[9] S. Chatterji, A discussion of the paper "Crystallisation in pores" by G.W. Scherer, Cement and Concrete Research 30 (2000) 669-671.

[10] L. Pel, H. Huinink, K. Kopinga, Ion transport and crystallization in inorganic building materials as studied by nuclear magnetic resonance, Applied Physics Letters 81 (2002) 2893-2895.

[11] U. Nachshon, E. Shahraeeni, D. Or, M. Dragila, N. Weisbrod, Infrared thermography of evaporative fluxes and dynamics of salt deposition on heterogeneous porous surfaces, Water Resources Research 47 (2011) W12519.

[12] N. Sghaier, M. Prat, Effect of efflorescence formation on drying kinetics of porous media, Transport in Porous Media 80 (3) (2009) 441-454.

[13] H. Eloukabi, N. Sghaier, M. Prat, S. Ben Nassrallah, Drying experiments in a hydrophobic model porous medium in the presence of a dissolved salt, Chemical Engineering and Technology 34 (7) (2011) 1085-1094.

[14] S. Veran-Tissoires, M. Marcoux, M. Prat, Discrete salt crystallization at the surface of a porous medium, Physical Review Letters 108 (2012) 054502 .

[15] S. Veran-Tissoires, M. Marcoux, M. Prat, Salt crystallization at the surface of a heterogeneous porous medium, Europhysics Letters 98 (2012) 34005

[16] M.N. Rad, N. Shokri, Nonlinear effects of salt concentrations on evaporation from porous media, Geophysical Research Letters 39 (2012) L04403.

[17] N. Sghaier, M. Prat, S. Ben Nasrallah, On the influence of sodium chloride concentration on equilibrium contact angle, Chemical Engineering Journal 122 (2006) 47-53.

[18] J. Van Brakel, Mass transfer in convective drying, in: A.S. Mujumdar (Ed.), Advances in Drying, Hemisphere, New York, 1980, pp. 217-267.

[19] Y. Le Bray, M. Prat, Three dimensional pore network simulation of drying in capillary porous media, International Journal of Heat and Mass Transfer 42 (1999) 4207-4224.

[20] P. Coussot, Scaling approach to the convective drying of a porous medium, European Physical Journal B: Condensed Matter and Complex Systems 15 (2000) 557-566

[21] J.H. Kim, A. Ochoa, S. Whitaker, Diffusion in anisotropic porous media, Transport in Porous Media 2 (1987) 327-356.

[22] R.D. Deegan, O. Bakajin, T.F. Dupont, G. Huber, S.R. Nagel, T.A. Witten, Capillary flow as the cause of ring stains from dried liquid drops, Nature 389 (1997) 827-829.

[23] H. Hu, R.G. Larson, Evaporation of a sessile droplet on a substrate, The Journal of Physical Chemistry B 106 (2002) 1334-1344.

[24] F.A.L. Dullien, Porous media: Fluid Transport and Pore Structure, Academic Press, 1992.

[25] R.A. Robinson, The vapour pressures of solutions of potassium chloride and sodium chloride, Transactions of the Royal Society of New Zealand 75 (2) (1945) 203-217.

[26] E.R. Washburn, The creeping of solutions, Journal of Physical Chemistry 31 (8) (1927) $1246-1248$.

[27] S. Veran-Tissoires, S. Geoffroy, M. Marcoux, M. Prat, Chapter 8 evaporation and wicking in porous materials, in: R. Masoodi, K.M. Pillai (Eds.), Traditional and Modern Modeling Approaches, Taylor and Francis, 2012.

[28] S. Veran-Tissoires, M. Marcoux, M. Prat, On the formation of patchy salt efflorescence at the evaporative surface of a porous medium, submitted for publication. 